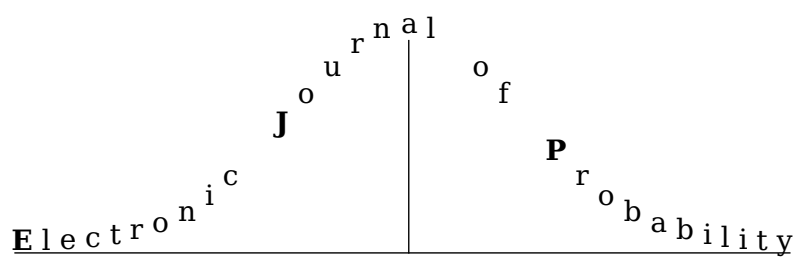

Electron. J. Probab. 25 (2020), no. 14, 1-24.

ISSN: 1083-6489 https://doi.org/10.1214/19-EJP405

\title{
Large deviations for the largest eigenvalue of the sum of two random matrices*
}

\author{
Alice Guionnet ${ }^{\dagger} \quad$ Mylène Maïda ${ }^{\ddagger}$
}

\begin{abstract}
In this paper, we consider the addition of two matrices in generic position, namely $A+U B U^{*}$, where $U$ is drawn under the Haar measure on the unitary or the orthogonal group. We show that, under mild conditions on the empirical spectral measures of the deterministic matrices $A$ and $B$, the law of the largest eigenvalue satisfies a large deviation principle, in the scale $N$, with an explicit rate function involving the limit of spherical integrals. We cover in particular the case when $A$ and $B$ have no outliers.
\end{abstract}

Keywords: random matrix; large deviations; extreme eigenvalues; free convolution. AMS MSC 2010: 15A52; 60F10; 46L54.

Submitted to EJP on November 23, 2018, final version accepted on December 22, 2019.

Supersedes arXiv: 1810.02538.

Supersedes HAL: hal -01887673.

\section{Introduction}

Understanding the spectrum of the sum $A+B$ of two Hermitian matrices knowing the spectra of $A$ and $B$ respectively is a classical and difficult problem. Since the pioneering works of Voiculescu [27], we know that free probability provides efficient tools to describe, at least asymptotically, the spectrum of the sum of two large Hermitian matrices in generic position from one another. More precisely, if $A_{N}$ and $B_{N}$ are two deterministic $N \times N$ Hermitian matrices and $U_{N}$ is a unitary random matrix distributed according to the Haar measure, then, in the large $N$ limit, $A_{N}$ and $U_{N} B_{N} U_{N}^{*}$ are asymptotically free and the spectral distribution of $H_{N}:=A_{N}+U_{N} B_{N} U_{N}^{*}$ is given by the free convolution of the spectral distributions of $A_{N}$ and $B_{N}$. This global law, that is the convergence of the spectral distribution of $H_{N}$ at macroscopic scale, has been studied in details in [26, 25] among others. The local law, that is the comparison of the spectral

\footnotetext{
*This work was supported by the LABEX MILYON (ANR-10-LABX-0070) of Université de Lyon within the program "Investissements d'Avenir" (ANR-11-IDEX- 0007) and by the Labex CEMPI (ANR-11-LABX-0007-01) of Université de Lille operated by the French National Research Agency (ANR).

†École Normale Supérieure de Lyon, France. E-mail: aguionne@umpa.ens - lyon . fr

${ }^{\ddagger}$ Université de Lille, France. E-mail: mylene.maida@univ-lille.fr
} 
distribution of $H_{N}$ with the free additive convolution of the spectral distributions of $A_{N}$ and $B_{N}$ below the macroscopic scale was then investigated in [22] and [4]. In this paper, we will be interested in the behavior of the largest eigenvalue of $H_{N}$. As a corollary of the results of [14] on strong asymptotic freeness, we know that if $A_{N}$ and $B_{N}$ have no outliers, then the largest eigenvalue of $H_{N}$ converges to the right edge of the support of the free convolution of the spectral distributions of $A_{N}$ and $B_{N}$. In this work, we investigate the large deviations of this extreme eigenvalue.

In the framework of random matrix theory, there are very few large deviation results known about the spectrum, basically because the eigenvalues are complicated functions of the entries. A notable exception is given by the Gaussian invariant ensembles for which the joint law of the eigenvalues can be explicitly written as a Coulomb gas. Based on this explicit formula, large deviation principles for the empirical spectral distribution at global scale have been established in [9] and for the largest eigenvalue in [8]. Another special case is given by the sum of a deterministic matrix and a Gaussian invariant ensemble. Then, the spectrum can be constructed as the realization at time one of a Hermitian (or symmetric) Brownian motion starting from a given deterministic matrix. This point of view was used by [21] to study the large deviations of the empirical measure, and the large deviations for the process of the largest eigenvalue starting from the origin were derived in [16]. One of the applications of the present paper is to provide the large deviations for the largest eigenvalue of this sum by using another approach based on spherical integrals. Beyond these cases where specific tools are available, it was observed by [12] that deviations of the spectrum of Wigner matrices for which the distribution of the entries has a tail which is heavier than Gaussian are naturally created by large entries. This key remark allowed to obtain the large deviations for the empirical measure in [12] (see also [18] for the counterpart for covariance matrices) and for the largest eigenvalue in [2]. Large deviations for the spectrum of Wigner matrices with subGaussian entries is still completely open as far as the empirical measure is concerned. One can mention the deviations results of [3] for the moments of the empirical spectral distribution in several models. Concerning the deviations of the largest eigenvalue, beyond the works $[8,16,2]$ already cited above, the following models have been so far studied : Gaussian ensembles plus a rank one perturbation by [23], very thin covariance matrices by [17], finite rank perturbations of deterministic matrices or unitarily invariant ensembles by [10]. In a companion paper [19], Guionnet and Husson have established a large deviation principle for the largest eigenvalue of Wigner matrices with entries having sharp sub-Gaussian tails, such as Rademacher matrices. They show that the speed and the rate function of this large deviation principle are the same as in the Gaussian case.

\section{Statement of the results}

Let $\left(A_{N}\right)_{N \geq 1}$ and $\left(B_{N}\right)_{N \geq 1}$ be two sequences of deterministic real diagonal matrices, with $A_{N}$ and $B_{N}$ of size $N \times N$. We denote by $\lambda_{1}^{\left(A_{N}\right)} \geq \ldots \geq \lambda_{N}^{\left(A_{N}\right)}$ and $\lambda_{1}^{\left(B_{N}\right)} \geq \ldots \geq \lambda_{N}^{\left(B_{N}\right)}$ their respective eigenvalues in non increasing order, by

$$
\left\|A_{N}\right\|:=\max \left(\left|\lambda_{1}^{\left(A_{N}\right)}\right|,\left|\lambda_{N}^{\left(A_{N}\right)}\right|\right) \text { and }\left\|B_{N}\right\|:=\max \left(\left|\lambda_{1}^{\left(B_{N}\right)}\right|,\left|\lambda_{N}^{\left(B_{N}\right)}\right|\right)
$$

their respective spectral radius. We define by

$$
\hat{\mu}_{A_{N}}:=\frac{1}{N} \sum_{j=1}^{N} \delta_{\lambda_{j}^{\left(A_{N}\right)}} \text { and } \hat{\mu}_{B_{N}}:=\frac{1}{N} \sum_{j=1}^{N} \delta_{\lambda_{j}^{\left(B_{N}\right)}}
$$

their respective empirical spectral distributions. 
For $\beta=1$ or 2 , we denote by $m_{N}^{\beta}$ the Haar measure on the orthogonal group $\mathcal{O}_{N}$ if $\beta=1$ and on the unitary group $\mathcal{U}_{N}$ if $\beta=2$. For any $N \times N$ unitary matrix $U$, we denote by $H_{N}(U):=A_{N}+U B_{N} U^{*}$ and by $\lambda_{\max }^{N}$ the largest eigenvalue of $H_{N}(U)$. The goal of the present work is to establish a large deviation principle for the law of $\lambda_{\max }^{N}$ under the Haar measure $m_{N}^{\beta}$. This large deviation principle holds under mild assumptions that we now detail.

\section{Assumption 2.1.}

( $\left.H_{\text {bulk }}\right)$ The sequences of empirical spectral distributions $\left(\hat{\mu}_{A_{N}}\right)_{N \geq 1}$ and $\left(\hat{\mu}_{B_{N}}\right)_{N \geq 1}$ converge weakly as $N$ grows to infinity respectively to $\mu_{a}$ and $\mu_{b}$, compactly supported on $\mathbb{R}$. Moreover, $\sup _{N \geq 1}\left(\left\|A_{N}\right\|+\left\|B_{N}\right\|\right)<\infty$.

( $\left.H_{\text {edge }}\right)$ The largest eigenvalues $\lambda_{1}^{\left(A_{N}\right)}$ and $\lambda_{1}^{\left(B_{N}\right)}$ converge as $N$ grows to infinity to $\rho_{a}$ and $\rho_{b}$ respectively.

A key argument of the proof will be a tilt of the measure by a rank one spherical integral. The rank one spherical integral is defined as follows: for any $\theta \geq 0$ and $M_{N}$ an Hermitian matrix of size $N$,

$$
I_{N}^{\beta}\left(\theta, M_{N}\right):=\int \mathrm{e}^{N \theta\left(U M_{N} U^{*}\right)_{11}} m_{N}^{\beta}(\mathrm{d} U) \quad \text { and } \quad J_{N}^{\beta}\left(\theta, M_{N}\right):=\frac{1}{N} \log I_{N}^{\beta}\left(\theta, M_{N}\right) .
$$

The rate function of our large deviation principle will crucially involve the limit $J_{\mu}^{\beta}(\theta, \rho)$ of $J_{N}^{\beta}\left(\theta, H_{N}\right)$ as $N$ grows to infinity, which we now describe. For $\mu$ a compactly supported probability measure on $\mathbb{R}$, we denote by $r(\mu)$ the right edge of the support of $\mu$ and by $G_{\mu}$ the Stieltjes transform of $\mu$ given for $\lambda>r(\mu)$ by

$$
G_{\mu}(\lambda):=\int \frac{1}{\lambda-y} \mu(\mathrm{d} y) .
$$

It is decreasing on the interval $(r(\mu), \infty)$. By taking the limit as $\lambda$ decreases to $r(\mu)$, one can also define $G_{\mu}(r(\mu)) \in \mathbb{R}_{+} \cup \infty$. As $G_{\mu}$ is bijective from $(r(\mu), \infty)$ to $\left(0, G_{\mu}(r(\mu))\right)$, one can define its inverse on the latter interval, that we denote by $K_{\mu}$. Then, for any $z \in\left(0, G_{\mu}(r(\mu))\right)$, we define

$$
R_{\mu}(z):=K_{\mu}(z)-\frac{1}{z}
$$

The function $R_{\mu}$ is called the $R$-transform of $\mu$. One can check that $R_{\mu}$ is an increasing function and that $\lim _{z \rightarrow 0} R_{\mu}(z)=\int \lambda \mu(\mathrm{d} \lambda): R_{\mu}$ is bijective from $\left(0, G_{\mu}(\mathrm{r}(\mu))\right)$ to $\left(\int \lambda \mu(\mathrm{d} \lambda), \mathrm{r}(\mu)-\frac{1}{G_{\mu}(\mathrm{r}(\mu))}\right)$. We denote by $Q_{\mu}$ its inverse on this interval. We can now define, for $\beta=1$ or $2, \theta \geq 0, \mu$ a compactly supported probability measure and $\rho \geq \mathrm{r}(\mu)$ :

$$
J_{\mu}^{\beta}(\theta, \rho):= \begin{cases}\frac{\beta}{2} \int_{0}^{\frac{2 \theta}{\beta}} R_{\mu}(u) \mathrm{d} u, & \text { if } 0 \leq \frac{2 \theta}{\beta} \leq G_{\mu}(\rho), \\ \theta \rho-\frac{\beta}{2} \log \theta-\frac{\beta}{2} \int \log (\rho-y) \mu(\mathrm{d} y)+\frac{\beta}{2}\left(\log \frac{\beta}{2}-1\right), & \text { if } \frac{2 \theta}{\beta}>G_{\mu}(\rho) .\end{cases}
$$

The convergence of $J_{N}^{\beta}\left(\theta, M_{N}\right)$ towards $J_{\mu}^{\beta}(\theta, \rho)$, obtained by the authors in [20], will be stated precisely in Lemma 3.4. At this point, we want to emphasize that, for $\theta$ large enough, the limit depends not only on the limiting spectral distribution $\mu$ but also of the limit $\rho$ of the largest eigenvalue of $M_{N}$ : this observation is crucial in our use of the spherical integral to produce an interesting tilt. If $\mu_{1}$ and $\mu_{2}$ are two probability measures compactly supported on $\mathbb{R}$, we denote by $\mu_{1} \boxplus \mu_{2}$ the free convolution of $\mu_{1}$ and $\mu_{2}$. It is uniquely determined as the unique probability measure with $R$-transform equal to the sum of the R-transforms of $\mu_{1}$ and $\mu_{2}$ (see [27]). For any $\theta \geq 0$ and $x \geq \mathrm{r}\left(\mu_{a} \boxplus \mu_{b}\right)$, we denote by

$$
I^{\beta}(\theta, x):=J_{\mu_{a} \boxplus \mu_{b}}^{\beta}(\theta, x)-J_{\mu_{a}}^{\beta}\left(\theta, \rho_{a}\right)-J_{\mu_{b}}^{\beta}\left(\theta, \rho_{b}\right),
$$


and

$$
I^{\beta}(x):= \begin{cases}\sup _{\theta \geq 0} I^{\beta}(\theta, x), & \text { if } x \geq \mathrm{r}\left(\mu_{a} \boxplus \mu_{b}\right) \\ +\infty, & \text { otherwise }\end{cases}
$$

It is easy to check the following:

Lemma 2.2. Let $\mu_{a}, \mu_{b}, \rho_{a}$ and $\rho_{b}$ be given as in Assumption 2.1. For $\beta=1$ or 2 , the function $I^{\beta}$ is a good rate function, that is for any $\alpha \in \mathbb{R}$, the level set $\left\{I^{\beta} \leq \alpha\right\}$ is a compact subset of $\mathbb{R}$. Moreover, for any $x>\rho_{a}+\rho_{b}, I^{\beta}(x)=+\infty$.

The proof will be given at the beginning of Section 4 . We can now state the main results of this paper. The first result is the following large deviation upper bound:

Proposition 2.3. Under Assumption 2.1, for $\beta=1$ or 2 , for any $x \in \mathbb{R}$ such that

$$
G_{\mu_{a} \boxplus \mu_{b}}(x) \leq \min \left(G_{\mu_{a}}\left(\rho_{a}\right), G_{\mu_{b}}\left(\rho_{b}\right)\right),
$$

we have

$$
\lim _{\delta \downarrow 0} \limsup _{N \rightarrow+\infty} \frac{1}{N} \log m_{N}^{\beta}\left(\lambda_{\max }^{N} \in[x-\delta, x+\delta]\right) \leq-I^{\beta}(x) .
$$

We will then derive the following large deviation lower bound:

Proposition 2.4. Under Assumption 2.1, for $\beta=1$ or 2 , for any $x \in \mathbb{R}$ such that

$$
G_{\mu_{a} \boxplus \mu_{b}}(x) \leq \min \left(G_{\mu_{a}}\left(\rho_{a}\right), G_{\mu_{b}}\left(\rho_{b}\right)\right),
$$

we have

$$
\lim _{\delta \downarrow 0} \liminf _{N \rightarrow+\infty} \frac{1}{N} \log m_{N}^{\beta}\left(\lambda_{\max }^{N} \in[x-\delta, x+\delta]\right) \geq-I^{\beta}(x) .
$$

This leads to the following important corollary:

Theorem 2.5. Under Assumption 2.1 and if moreover,

$$
G_{\mu_{a} \boxplus \mu_{b}}\left(r\left(\mu_{a} \boxplus \mu_{b}\right)\right) \leq \min \left(G_{\mu_{a}}\left(\rho_{a}\right), G_{\mu_{b}}\left(\rho_{b}\right)\right) ，
$$

(NoOut)

then, for $\beta=1$ or 2 , the law of $\lambda_{\max }^{N}$ under $m_{N}^{\beta}$ satisfies a large deviation principle in the scale $N$ with good rate function $I^{\beta}$. More precisely, for any $F$ closed Borel subset of $\mathbb{R}$,

$$
\limsup _{N \rightarrow+\infty} \frac{1}{N} \log m_{N}^{\beta}\left(\lambda_{\max }^{N} \in F\right) \leq-\inf _{F} I^{\beta}
$$

and for any $O$ open Borel subset of $\mathbb{R}$,

$$
\liminf _{N \rightarrow+\infty} \frac{1}{N} \log m_{N}^{\beta}\left(\lambda_{\max }^{N} \in 0\right) \geq-\inf _{O} I^{\beta}
$$

A few remarks have to be made on the condition (NoOut). Under assumptions that are slightly stronger than Assumption $\left(H_{\text {bulk }}\right)$, [7] established that, whenever (NoOut) is satisfied, $A_{N}+U B_{N} U^{*}$ has no outlier, that is, its largest eigenvalue converges to $\mathrm{r}\left(\mu_{a} \boxplus \mu_{b}\right)$. Another related remark is that, if $A_{N}$ and $B_{N}$ have no outliers, namely $\rho_{a}=\mathrm{r}\left(\mu_{a}\right)$ and $\rho_{b}=\mathrm{r}\left(\mu_{b}\right)$, then the condition (NoOut) is automatically satisfied. This will be stated in Lemma 6.1 and leads to the following corollary

Corollary 2.6. Under the assumption ( $\left.H_{\mathrm{bulk}}\right)$, if $A_{N}$ and $B_{N}$ have no outliers, then for $\beta=1$ or 2 , the law of $\lambda_{\max }^{N}$ under $m_{N}^{\beta}$ satisfies a large deviation principle in the scale $N$ with good rate function $I^{\beta}$.

From there, one can recover partly Theorem 3.2. in [23]. 
Remark 2.7. If we choose $A_{N}$ to be a rank one deterministic matrix with eigenvalue $\rho_{a}>0$ and $U B_{N} U^{*}$ to be a random matrix from the Gaussian Unitary (or Orthogonal) Ensemble, one can study the largest eigenvalue of $A_{N}+U B_{N} U^{*}$ by conditioning on the deviations of the largest eigenvalue of $U B_{N} U^{*}$. These large deviations were obtained in [8] and we denote by $J^{\beta}$ its rate function. If $\rho_{a} \leq \sqrt{\frac{\beta}{2}}$, we know that the deformed model has no outliers and one can apply Corollary 5 . For any $x \geq \sqrt{2 \beta}$, the rate function of the deformed model is given by $K^{\beta}(x):=\inf _{\sqrt{2 \beta} \leq y \leq x}\left(J^{\beta}(y)+I^{\beta}(x)\right)$, where $I^{\beta}$ corresponds to $\mu_{a}=\delta_{0}, \mu_{b}=\sigma_{\beta}$ and $\rho_{b}=y$. Standard computations allow to identify the rate function as the funtion $K_{\rho_{a}}^{\beta}$ in [23].

To get a taste of what happens in the case with outliers, we also consider in Appendix A the following model: let $\left(U_{1}^{(1)}, \ldots, U_{1}^{(p)}\right)$ be independent random vectors uniformly distributed on the unit sphere (in $\mathbb{R}^{N}$ if $\beta=1$ and $\mathbb{C}^{N}$ if $\beta=2$ ) and $\gamma_{1}, \ldots, \gamma_{p}$ be nonnegative real numbers. We consider the following deformed model :

$$
X_{N}:=A_{N}+U B_{N} U^{*}+\sum_{i=1}^{p} \gamma_{i} U_{1}^{(i)}\left(U_{1}^{(i)}\right)^{*}
$$

We show in Theorem A.5 that we still have a large deviation principle, for which the rate function will depend on the $\gamma_{i}$ 's. The rest of the paper will be organized as follows: in the next section, we will first prove a more general result than Proposition 2.3, that holds not only for $m_{N}^{\beta}$ but also for a whole family of tilted measures. This will be helpful in the proof of Proposition 2.4, that will be developed in Section 5. Before getting there, we will study in Section 4 some properties of the rate function $I^{\beta}$. The last section will be devoted to the proof of Theorem 2.5 and Corollary 2.6, with Lemma 6.1 as prerequisite. At the end of the paper, in Appendix A, we will study the deviations of the largest eigenvalue of $X_{N}$ for the deformed model (2.4).

\section{Large deviation upper bound for tilted measures}

For $\theta \geq 0, \beta=1$ or 2 , we define a tilted measure on $\mathcal{O}_{N}$ if $\beta=1$ and $\mathcal{U}_{N}$ if $\beta=2$ as follows

$$
m_{N}^{\beta, \theta}(\mathrm{d} U):=\frac{I_{N}^{\beta}\left(\theta, A_{N}+U B_{N} U^{*}\right)}{I_{N}^{\beta}\left(\theta, A_{N}\right) I_{N}^{\beta}\left(\theta, B_{N}\right)} m_{N}^{\beta}(\mathrm{d} U) .
$$

It is easy to check that $m_{N}^{\beta, \theta}$ is a probability measure: indeed, for any $U$, we have that $I_{N}^{\beta}\left(\theta, A_{N}+U B_{N} U^{*}\right) \geq 0$ and $\mathbb{E}_{m^{\beta}}\left(I_{N}^{\beta}\left(\theta, A_{N}+U B_{N} U^{*}\right)\right)=I_{N}^{\beta}\left(\theta, A_{N}\right) I_{N}^{\beta}\left(\theta, B_{N}\right)$. For these tilted measures, we have the following weak large deviation upper bound:

Proposition 3.1. Under Assumption 2.1, for $\beta=1$ or 2 , for any $\theta \geq 0$, for any $x<$ $r\left(\mu_{a} \boxplus \mu_{b}\right)$,

$$
\lim _{\delta \downarrow 0} \limsup _{N \rightarrow+\infty} \frac{1}{N} \log m_{N}^{\beta, \theta}\left(\lambda_{\max }^{N} \in[x-\delta, x+\delta]\right)=-\infty
$$

and for any $x \geq r\left(\mu_{a} \boxplus \mu_{b}\right)$ such that

$$
G_{\mu_{a} \boxplus \mu_{b}}(x) \leq \min \left(G_{\mu_{a}}\left(\rho_{a}\right), G_{\mu_{b}}\left(\rho_{b}\right)\right),
$$

we have,

$$
\lim _{\delta \downarrow 0} \limsup _{N \rightarrow+\infty} \frac{1}{N} \log m_{N}^{\beta, \theta}\left(\lambda_{\max }^{N} \in[x-\delta, x+\delta]\right) \leq-\left[I^{\beta}(x)-I^{\beta}(\theta, x)\right] .
$$

Remark 3.2. Applying this proposition with $\theta=0$ gives Proposition 2.3. 
As we will see in Section 5, establishing an upper bound for any $\theta \geq 0$ will be useful in the proof of Proposition 2.4. To prove Proposition 3.1, and in particular its first statement, we will need to check that, under $m_{N}^{\beta, \theta}$ the empirical spectral distribution

$$
\hat{\mu}_{N}:=\frac{1}{N} \sum_{j=1}^{N} \delta_{\lambda_{j}^{\left(H_{N}(U)\right)}}
$$

of $H_{N}(U)=A_{N}+U B_{N} U^{*}$ concentrates around the deterministic probability measure

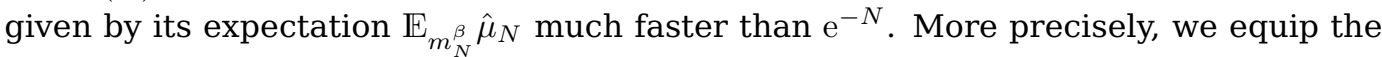
set $\mathcal{P}(\mathbb{R})$ of probability measures on $\mathbb{R}$ with the bounded Lipschitz distance $\mathrm{d}$ : for any Lipschitz function $f: \mathbb{R} \rightarrow \mathbb{R}$, we define $\|f\|_{\infty}:=\sup _{x \in \mathbb{R}}|f(x)|$ and $\|f\|_{\text {Lip }}:=$ $\sup _{x \neq y} \frac{|f(x)-f(y)|}{|x-y|}$, then for any $\mu$ and $\nu$ in $\mathcal{P}(\mathbb{R})$,

$$
\mathrm{d}(\mu, \nu):=\sup _{\substack{\|f\|_{\infty} \leq 1 \\\|f\|_{\text {Lip }} \leq 1}}\left(\int f \mathrm{~d} \mu-\int f \mathrm{~d} \nu\right) .
$$

We then have the following concentration result:

Lemma 3.3. Under Assumption ( $\left.H_{\mathrm{bulk}}\right)$, for $\beta=1$ or 2 and any $\theta \geq 0$,

$$
\limsup _{N \rightarrow \infty} \frac{1}{N} \log m_{N}^{\beta, \theta}\left(\mathrm{d}\left(\hat{\mu}_{N}, \mathbb{E}_{m_{N}^{\beta}} \hat{\mu}_{N}\right)>N^{-1 / 4}\right)=-\infty .
$$

Proof. Let $\beta=1$ or 2 and $\theta \geq 0$ be fixed. Observe that for any Hermitian matrix $M_{N}$ bounded by $K$ in operator norm we have

$$
e^{-\theta K N} \leq I_{N}^{\beta}\left(\theta, A_{N}\right) \leq e^{\theta K N}
$$

As a consequence, for any Borel subset $A$ of $\mathcal{O}_{N}$ if $\beta=1$ and $\mathcal{U}_{N}$ if $\beta=2$, we have:

$$
\begin{aligned}
m_{N}^{\beta, \theta}(A) & =\frac{1}{I_{N}^{\beta}\left(\theta, A_{N}\right) I_{N}^{\beta}\left(\theta, B_{N}\right)} \int_{A} I_{N}^{\beta}\left(\theta, A_{N}+U B_{N} U^{*}\right) m_{N}^{\beta}(\mathrm{d} U) \\
& \leq \mathrm{e}^{2 N \theta K} m_{N}^{\beta}(A),
\end{aligned}
$$

with $K:=\sup _{N \geq 1}\left(\left\|A_{N}\right\|+\left\|B_{N}\right\|\right)$, which is assumed to be finite. Therefore it is enough to prove Lemma 3.3 for $\theta=0$, that is

$$
\limsup _{N \rightarrow \infty} \frac{1}{N} \log m_{N}^{\beta}\left(\mathrm{d}\left(\hat{\mu}_{N}, \mathbb{E}_{m_{N}^{\beta}} \hat{\mu}_{N}\right)>N^{-1 / 4}\right)=-\infty .
$$

For $\beta=2$, Theorem 3.8 in [24] states that there exist $c, C>0$ such that

$$
m_{N}^{2}\left(\mathrm{~d}\left(\hat{\mu}_{N}, \mathbb{E}_{m_{N}^{2}} \hat{\mu}_{N}\right)>N^{-1 / 4}\right) \leq C \mathrm{e}^{-c N^{3 / 2}},
$$

from which the lemma follows. A careful reading of [24] shows that the exact same result as (3.4) also holds for $\beta=1$.

Before proving Proposition 3.1, we will recall some results about the convergence and the continuity of spherical integrals.

Lemma 3.4 (Proposition 2.1 in [23] and Theorem 6 in [20]). For any $\theta \geq 0$, there exists a continuous function $g_{\theta}$ with $g_{\theta}(0)=0$ such that for any $\delta>0$, if the sequences $\left(G_{N}\right)_{N \geq 1}$ and $\left(G_{N}^{\prime}\right)_{N \geq 1}$ are such that $\sup _{N}\left(\left\|G_{N}\right\|+\left\|G_{N}^{\prime}\right\|\right)<\infty$, and for $N$ large enough, $\mathrm{d}\left(\hat{\mu}_{G_{N}}, \hat{\mu}_{G_{N}^{\prime}}\right) \leq N^{-1 / 4}$ and $\left|\lambda_{1}\left(G_{N}\right)-\lambda_{1}\left(G_{N}^{\prime}\right)\right| \leq \delta$, then,

$$
\left|\frac{1}{N} \log I_{N}^{\beta}\left(\theta, G_{N}\right)-\frac{1}{N} \log I_{N}^{\beta}\left(\theta, G_{N}^{\prime}\right)\right| \leq g_{\theta}(\delta) .
$$


If moreover, $\hat{\mu}_{G_{N}}$ converges weakly, as $N$ goes to infinity to $\mu$ and $\lambda_{1}\left(G_{N}\right)$ converges to $\rho$, then

$$
\frac{1}{N} \log I_{N}^{\beta}\left(\theta, G_{N}\right) \underset{N \rightarrow \infty}{\longrightarrow} J_{\mu}(\theta, \rho) .
$$

We can now prove Proposition 3.1. In the sequel, we will denote by $\nu_{N}^{\beta}:=\mathbb{E}_{m_{N}^{\beta}} \hat{\mu}_{N}$.

Proof of Proposition 3.1. The first claim (3.1) is a direct consequence of the previous lemma. Indeed, let $x<\mathrm{r}\left(\mu_{a} \boxplus \mu_{b}\right)$ and $\delta_{0}:=\frac{\mathrm{r}\left(\mu_{a} \boxplus \mu_{b}\right)-x}{2}$. Then, for any $\delta \leq \delta_{0}$, there exists $\varepsilon(\delta)>0$,

$$
\left\{\lambda_{\max }^{N} \in[x-\delta, x+\delta]\right\} \subset\left\{\mathrm{d}\left(\hat{\mu}_{N}, \mu_{a} \boxplus \mu_{b}\right)>\varepsilon(\delta)\right\} .
$$

Using Corollary 5.4.11 for $\beta=2$ and Exercise 5.4.18 for $\beta=1$ in [1], we know that $\nu_{N}^{\beta}$ converges weakly to $\mu_{a} \boxplus \mu_{b}$ as $N$ goes to infinity. As the distance d metrizes the weak convergence, for $N$ large enough,

$$
\left\{\lambda_{\max }^{N} \in[x-\delta, x+\delta]\right\} \subset\left\{\mathrm{d}\left(\hat{\mu}_{N}, \nu_{N}^{\beta}\right)>\varepsilon(\delta) / 2\right\}
$$

so that, by Lemma 3.3, for any $\delta \leq \delta_{0}$,

$$
\limsup _{N \rightarrow \infty} \frac{1}{N} \log m_{N}^{\beta, \theta}\left(\lambda_{\max }^{N} \in[x-\delta, x+\delta]\right)=-\infty .
$$

We now prove (3.3). Let $\delta>0$ and $x \geq \mathrm{r}\left(\mu_{a} \boxplus \mu_{b}\right)$ be fixed and define the following event:

$$
\mathrm{E}_{N, \delta}^{x}:=\left\{\lambda_{\max }^{N} \in[x-\delta, x+\delta], \mathrm{d}\left(\hat{\mu}_{N}, \nu_{N}^{\beta}\right) \leq N^{-1 / 4}\right\}
$$

Then we have,

$$
m_{N}^{\beta, \theta}\left(\lambda_{\max }^{N} \in[x-\delta, x+\delta]\right) \leq m_{N}^{\beta, \theta}\left(\mathrm{E}_{N, \delta}^{x}\right)+m_{N}^{\beta, \theta}\left(\mathrm{d}\left(\hat{\mu}_{N}, \nu_{N}^{\beta}\right)>N^{-1 / 4}\right) .
$$

By Lemma 3.3, it is therefore enough to show that

$$
\lim _{\delta \downarrow 0} \limsup _{N \rightarrow \infty} \frac{1}{N} \log m_{N}^{\beta, \theta}\left(\mathrm{E}_{N, \delta}^{x}\right) \leq-\left[I^{\beta}(x)-I^{\beta}(\theta, x)\right] .
$$

To lighten a bit the notations we write $A, B$ and $H$ for $A_{N}, B_{N}$ and $H_{N}=A_{N}+U B_{N} U^{*}$ respectively. For any $\theta, \theta^{\prime} \geq 0$, we have

$$
\begin{aligned}
m_{N}^{\beta, \theta}\left(\mathrm{E}_{N, \delta}^{x}\right) & =\frac{1}{I_{N}^{\beta}(\theta, A) I_{N}^{\beta}(\theta, B)} \mathbb{E}_{m_{N}^{\beta}}\left(1_{\mathrm{E}_{N, \delta}^{x}} I_{N}^{\beta}(\theta, H) \frac{I_{N}^{\beta}\left(\theta^{\prime}, H\right)}{I_{N}^{\beta}\left(\theta^{\prime}, H\right)}\right) \\
& \leq \frac{\mathrm{E}_{m_{N}^{\beta}}\left(I_{N}^{\beta}\left(\theta^{\prime}, H\right)\right)}{I_{N}^{\beta}(\theta, A) I_{N}^{\beta}(\theta, B)} \sup _{U \in \mathrm{E}_{N, \delta}^{x}} \frac{I_{N}^{\beta}\left(\theta, A+U B U^{*}\right)}{I_{N}^{\beta}\left(\theta^{\prime}, A+U B U^{*}\right)} \\
& =\frac{I_{N}^{\beta}\left(\theta^{\prime}, A\right) I_{N}^{\beta}\left(\theta^{\prime}, B\right)}{I_{N}^{\beta}(\theta, A) I_{N}^{\beta}(\theta, B)} \sup _{U \in \mathrm{E}_{N, \delta}^{x}} \frac{I_{N}^{\beta}\left(\theta, A+U B U^{*}\right)}{I_{N}^{\beta}\left(\theta^{\prime}, A+U B U^{*}\right)}
\end{aligned}
$$

We now have to estimate $\sup _{U \in \mathrm{E}_{N, \delta}^{x}} I_{N}^{\beta}\left(\theta, A+U B U^{*}\right)$ and $\inf _{U \in \mathrm{E}_{N, \delta}^{x}} I_{N}^{\beta}\left(\theta^{\prime}, A+U B U^{*}\right)$ respectively. We detail the first term, the second being similar. According to [7] (see also Section 4.1.2 in [13]), if $x \geq \mathrm{r}\left(\mu_{a} \boxplus \mu_{b}\right)$ satisfies $G_{\mu_{a} \boxplus \mu_{b}}(x) \leq \min \left(G_{\mu_{a}}\left(\rho_{a}\right), G_{\mu_{b}}\left(\rho_{b}\right)\right)$, then $m_{N}^{\beta}$ almost surely, $\lim \sup _{N \rightarrow \infty} \lambda_{\max }^{N} \leq x$ and therefore $\lim \sup _{N \rightarrow \infty} \mathrm{r}\left(\nu_{N}^{\beta}\right) \leq x$. It is therefore possible to build a sequence $\left(G_{N}\right)_{N \geq 1}$ of deterministic matrices such that $\sup _{N \geq 1}\left\|G_{N}\right\|<\infty$ and for any $N \geq 1, \lambda_{1}^{\left(G_{N}\right)}$ converges to $x$ and $\mathrm{d}\left(\hat{\mu}_{G_{N}}, \nu_{N}^{\beta}\right) \leq N^{-1 / 4}$. With the notations of Lemma 3.4, for $N$ large enough and for any $U \in \mathrm{E}_{N, \delta}^{x}$,

$$
\left|\frac{1}{N} \log I_{N}^{\beta}\left(\theta, A+U B U^{*}\right)-\frac{1}{N} \log I_{N}^{\beta}\left(\theta, G_{N}\right)\right| \leq g_{\theta}(\delta) .
$$


Therefore,

$$
\begin{aligned}
\limsup _{N \rightarrow \infty} \frac{1}{N} \log m_{N}^{\beta, \theta}\left(\mathrm{E}_{N, \delta}^{x}\right) & \leq \lim _{N \rightarrow \infty}\left(J_{N}^{\beta}\left(\theta^{\prime}, A\right)+J_{N}^{\beta}\left(\theta^{\prime}, B\right)-J_{N}^{\beta}(\theta, A)-J_{N}^{\beta}(\theta, B)\right) \\
& +\lim _{N \rightarrow \infty}\left(J_{N}^{\beta}\left(\theta, G_{N}\right)-J_{N}^{\beta}\left(\theta^{\prime}, G_{N}\right)\right)+g_{\theta}(\delta)+g_{\theta^{\prime}}(\delta) \\
& \leq-\left(I^{\beta}\left(\theta^{\prime}, x\right)-I^{\beta}(\theta, x)\right)+g_{\theta}(\delta)+g_{\theta^{\prime}}(\delta)
\end{aligned}
$$

where at the last line, we have used the second part of Lemma 3.4. Letting $\delta$ going to zero and then optimizing over $\theta^{\prime} \geq 0$, we get the required upper bound.

\section{Properties of the rate function $I^{\beta}$}

We now check the properties of the rate function $I^{\beta}$ defined in (2.1).

Proof of Lemma 2.2. An ingredient for the proof is the following: for any compactly supported $\mu$, for any $\theta \geq 0$ and $\rho \geq \operatorname{r}(\mu)$ such that $\theta \leq G_{\mu}(\rho)$, we have

$$
\rho-\frac{1}{\theta} \leq R_{\mu}(\theta) \leq \rho-\frac{1}{G_{\mu}(\rho)} .
$$

Indeed, as $K_{\mu}$ is a decreasing function, we have $R_{\mu}(\theta)=K_{\mu}(\theta)-\frac{1}{\theta} \geq \rho-\frac{1}{\theta}$. On the other hand, the limit of $R_{\mu}(\theta)$ as $\theta$ grows to $G_{\mu}(\rho)$ is $\rho-\frac{1}{G_{\mu}(\rho)}$. As $R_{\mu}$ is nondecreasing, we get the upper bound. Moreover, it is easy to check that, for any $x \geq 0$, there exist $C, C^{\prime} \in \mathbb{R}$ (depending on $\mu$ and $x$ but not on $\theta$ ) such that, for $\theta$ large enough, we have

$$
\theta x-\frac{\beta}{2} \log \theta+C \leq J_{\mu}^{\beta}(\theta, x) \leq \theta x+C^{\prime},
$$

so that, for any $x \geq 0$, there exists $c, c^{\prime} \in \mathbb{R}$ such that, for $\theta$ large enough,

$$
\theta\left(x-\rho_{a}-\rho_{b}\right)-\frac{\beta}{2} \log \theta+c \leq I^{\beta}(\theta, x) \leq \theta\left(x-\rho_{a}-\rho_{b}\right)+\beta \log \theta+c^{\prime} .
$$

If $x>\rho_{a}+\rho_{b}$, letting $\theta$ grow to infinity, we obtain that $I^{\beta}(x)=+\infty$.

If $\theta \geq 0$ is small enough,

$$
I^{\beta}(\theta, x)=\frac{\beta}{2} \int_{0}^{\frac{2 \theta}{\beta}}\left(R_{\mu_{a} \boxplus \mu_{b}}(u)-R_{\mu_{a}}(u)-R_{\mu_{b}}(u)\right) \mathrm{d} u=0,
$$

by the properties of the $R$-transform. The function $I^{\beta}$ is therefore nonnegative. If we denote by $g$ the lower semi-continuous function which is equal to $-\infty$ on $\left[\mathrm{r}\left(\mu_{a} \boxplus \mu_{b}\right),+\infty\right)$ and $+\infty$ outside, then $I^{\beta}=\sup \left(g, \sup _{\theta} I^{\beta}(\theta, \cdot)\right)$ is lower semi-continuous as a supremum of lower semi-continuous functions. As it is infinite outside the interval $\left[\mathrm{r}\left(\mu_{a} \boxplus \mu_{b}\right), \rho_{a}+\rho_{b}\right]$, it is a good rate function.

We will now turn to the proof of the lower bound of our large deviation principle, stated in Proposition 2.4. To complete its proof, we will need to further study the properties of the function $I^{\beta}$. First, let us remark that the cases when $\mu_{a}$ is a Dirac mass at $\rho_{a}$ (or $\mu_{b}$ is a Dirac mass at $\rho_{b}$ ) are not very interesting. In this case, the free convolution $\mu_{a} \boxplus \mu_{b}$ is just a shift of $\mu_{b}$ by $\rho_{a}$ (or respectively of $\mu_{a}$ by $\rho_{b}$ ) and $\lambda_{\max }^{N}$ converges with probability one to $\rho_{a}+\rho_{b}$. Hence, the large deviations have an infinite rate function in the scale $N$ except at $\rho_{a}+\rho_{b}$ where it vanishes.

Consequently, in the sequel, one can assume without loss of generality that

Assumption 4.1. $\mu_{a}$ is not a Dirac mass at $\rho_{a}$ and $\mu_{b}$ is not a Dirac mass at $\rho_{b}$. 
We have the following:

Lemma 4.2. Under Assumptions 2.1 and 4.1, for any $r\left(\mu_{a} \boxplus \mu_{b}\right) \leq x<\rho_{a}+\rho_{b}$ such that

$$
G_{\mu_{a} \boxplus \mu_{b}}(x) \leq \min \left(G_{\mu_{a}}\left(\rho_{a}\right), G_{\mu_{b}}\left(\rho_{b}\right)\right),
$$

then, for $\beta=1$ or 2 , there exists a unique $\theta \geq 0$ such that

$$
I^{\beta}(\theta, x)=\sup _{\theta^{\prime} \geq 0} I^{\beta}\left(\theta^{\prime}, x\right) .
$$

We denote by $\theta_{x}^{\beta}:=\operatorname{argmax}_{\theta \geq 0} I^{\beta}(\theta, x)$. For any $r\left(\mu_{a} \boxplus \mu_{b}\right) \leq y \leq \rho_{a}+\rho_{b}$ such that $x \neq y$,

$$
\sup _{\theta \geq 0} I^{\beta}(\theta, y)>I^{\beta}\left(\theta_{x}^{\beta}, y\right) .
$$

Proof of Lemma 4.2. Let $\mathrm{r}\left(\mu_{a} \boxplus \mu_{b}\right) \leq x<\rho_{a}+\rho_{b}$ such that

$$
G_{\mu_{a} \boxplus \mu_{b}}(x) \leq \min \left(G_{\mu_{a}}\left(\rho_{a}\right), G_{\mu_{b}}\left(\rho_{b}\right)\right) .
$$

The first remark is that if $G_{\mu_{a}}\left(\rho_{a}\right)$ and $G_{\mu_{b}}\left(\rho_{b}\right)$ are infinite, then $r\left(\mu_{a} \boxplus \mu_{b}\right)=\rho_{a}+\rho_{b}$ and there is nothing to check since $\left[r\left(\mu_{a} \boxplus \mu_{b}\right), \rho_{a}+\rho_{b}\right)$ is empty. Indeed, if $G_{\mu_{a}}\left(\rho_{a}\right)=$ $G_{\mu_{b}}\left(\rho_{b}\right)=\infty$, we see by the inequalities (4.1), that

$$
\lim _{x \rightarrow \infty} R_{\mu_{a}}(x)=\rho_{a} \quad \text { and } \quad \lim _{x \rightarrow \infty} R_{\mu_{b}}(x)=\rho_{b},
$$

so that

$$
\lim _{x \rightarrow \infty} K_{\mu_{a} \boxplus \mu_{b}}(x)=\rho_{a}+\rho_{b} \quad \text { and } \quad \lim _{x \rightarrow \rho_{a}+\rho_{b}} G_{\mu_{a} \boxplus \mu_{b}}(x)=\infty,
$$

leading to $r\left(\mu_{a} \boxplus \mu_{b}\right) \geq \rho_{a}+\rho_{b}$. By symmetry of the problem, without loss of generality, one can now assume that $G_{\mu_{a}}\left(\rho_{a}\right) \leq G_{\mu_{b}}\left(\rho_{b}\right)$ and $G_{\mu_{a}}\left(\rho_{a}\right)<\infty$.

With the function $I^{\beta}$ defined in (2.1), if we denote by $I_{x}^{\beta}$ the function $\theta \mapsto I^{\beta}(\theta, x)$, then there exist some constants $C_{1}, C_{2}$ and $C_{3}$ (that may depend on $\mu_{a}, \rho_{a}, \mu_{b}, \rho_{b}$ and $x$ but not on $\theta$ ) such that

$$
I_{x}^{\beta}(\theta)= \begin{cases}0, & \text { if } 0 \leq \frac{2 \theta}{\beta} \leq G_{\mu_{a} \boxplus \mu_{b}}(x), \\ \theta x-\frac{\beta}{2} \log \theta-\frac{\beta}{2} \int_{0}^{\frac{2 \theta}{\beta}}\left(R_{\mu_{a}}+R_{\mu_{b}}\right)(u) \mathrm{d} u+C_{1}, & \text { if } G_{\mu_{a}} \boxplus \mu_{b}(x) \leq \frac{2 \theta}{\beta} \leq G_{\mu_{a}}\left(\rho_{a}\right), \\ \theta\left(x-\rho_{a}\right)-\frac{\beta}{2} \int_{0}^{\frac{2 \theta}{\beta}} R_{\mu_{b}}(u) \mathrm{d} u+C_{2}, & \text { if } G_{\mu_{a}}\left(\rho_{a}\right) \leq \frac{2 \theta}{\beta} \leq G_{\mu_{b}}\left(\rho_{b}\right), \\ \theta\left(x-\rho_{a}-\rho_{b}\right)+\frac{\beta}{2} \log \theta+C_{3}, & \text { if } \frac{2 \theta}{\beta} \geq G_{\mu_{b}}\left(\rho_{b}\right),\end{cases}
$$

where the last line does not occur if $G_{\mu_{b}}\left(\rho_{b}\right)=\infty$. In the computation, we have used the well known fact that $R_{\mu_{a} \boxplus \mu_{b}}=R_{\mu_{a}}+R_{\mu_{b}}$ when the three functions are well defined. Therefore, one can check that the function $I_{x}^{\beta}$ is continuously differentiable and its derivative is given by:

$$
\left(I_{x}^{\beta}\right)^{\prime}(\theta)= \begin{cases}0, & \text { if } 0 \leq \frac{2 \theta}{\beta} \leq G_{\mu_{a} \boxplus \mu_{b}}(x), \\ x-K_{\mu_{a} \boxplus \mu_{b}}\left(\frac{2 \theta}{\beta}\right), & \text { if } G_{\mu_{a} \boxplus \mu_{b}}(x) \leq \frac{2 \theta}{\beta} \leq G_{\mu_{a}}\left(\rho_{a}\right), \\ x-\rho_{a}-R_{\mu_{b}}\left(\frac{2 \theta}{\beta}\right), & \text { if } G_{\mu_{a}}\left(\rho_{a}\right) \leq \frac{2 \theta}{\beta} \leq G_{\mu_{b}}\left(\rho_{b}\right), \\ x-\rho_{a}-\rho_{b}+\frac{\beta}{2 \theta}, & \text { if } \frac{2 \theta}{\beta} \geq G_{\mu_{b}}\left(\rho_{b}\right) .\end{cases}
$$

We now set $\alpha_{x}:=\frac{1}{\rho_{a}+\rho_{b}-x}$. We claim that

$$
\alpha_{x} \geq G_{\mu_{a}}\left(\rho_{a}\right)
$$


Indeed, $K_{\mu_{b}}$ is well defined on the interval $\left(0, G_{\mu_{b}}\left(\rho_{b}\right)\right)$, so that $K_{\mu_{b}}\left(G_{\mu_{a}}\left(\rho_{a}\right)\right)$ and therefore $K_{\mu_{a} \boxplus \mu_{b}}\left(G_{\mu_{a}}\left(\rho_{a}\right)\right)$ are well defined. As $K_{\mu_{a} \boxplus \mu_{b}}$ is a decreasing function and

$$
G_{\mu_{a} \boxplus \mu_{b}}(x) \leq G_{\mu_{a}}\left(\rho_{a}\right)
$$

we have

$$
x \geq K_{\mu_{a} \boxplus \mu_{b}}\left(G_{\mu_{a}}\left(\rho_{a}\right)\right)=K_{\mu_{a}}\left(G_{\mu_{a}}\left(\rho_{a}\right)\right)+K_{\mu_{b}}\left(G_{\mu_{a}}\left(\rho_{a}\right)\right)-\frac{1}{G_{\mu_{a}}\left(\rho_{a}\right)}
$$

As $K_{\mu_{b}}$ is also a decreasing function, this yields:

$$
x \geq K_{\mu_{a}}\left(G_{\mu_{a}}\left(\rho_{a}\right)\right)+K_{\mu_{b}}\left(G_{\mu_{b}}\left(\rho_{b}\right)\right)-\frac{1}{G_{\mu_{a}}\left(\rho_{a}\right)}=\rho_{a}+\rho_{b}-\frac{1}{G_{\mu_{a}}\left(\rho_{a}\right)},
$$

which is equivalent to $\alpha_{x} \geq G_{\mu_{a}}\left(\rho_{a}\right)$. There are therefore two cases to consider and we claim that:

Case 1: If $G_{\mu_{a}}\left(\rho_{a}\right) \leq \alpha_{x}<G_{\mu_{b}}\left(\rho_{b}\right)$, then $I_{x}^{\beta}$ reaches its maximum at

$$
\theta_{x}^{\beta}:=\frac{\beta}{2} Q_{\mu_{b}}\left(x-\rho_{a}\right),
$$

where $Q_{\mu_{b}}$ is the inverse of $R_{\mu_{b}}$ as defined in Section 2;

Case 2: If $\alpha_{x} \geq G_{\mu_{b}}\left(\rho_{b}\right)$, then $I_{x}^{\beta}$ reaches its maximum at $\theta_{x}^{\beta}:=\frac{\beta}{2} \alpha_{x}$.

Let us now prove this claim. On the interval $\left[0, \frac{\beta}{2} G_{\mu_{a}}\left(\rho_{a}\right)\right]$, the function $\left(I_{x}^{\beta}\right)^{\prime}$ is nondecreasing and it vanishes at zero, it is therefore nonnegative so that $I_{x}^{\beta}$ is nondecreasing on this interval. We have

$$
\left(I_{x}^{\beta}\right)^{\prime}\left(\frac{\beta}{2} G_{\mu_{a}}\left(\rho_{a}\right)\right) \geq 0 \quad \text { and } \quad\left(I_{x}^{\beta}\right)^{\prime}\left(\frac{\beta}{2} G_{\mu_{b}}\left(\rho_{b}\right)\right)=-\frac{1}{\alpha_{x}}+\frac{1}{G_{\mu_{b}}\left(\rho_{b}\right)} .
$$

Moreover, as $R_{\mu_{b}}$ is increasing, $\left(I_{x}^{\beta}\right)^{\prime}$ is decreasing on the interval $\left[\frac{\beta}{2} G_{\mu_{a}}\left(\rho_{a}\right), \frac{\beta}{2} G_{\mu_{b}}\left(\rho_{b}\right)\right]$. We now distinguish the two cases.

In Case $1,\left(I_{x}^{\beta}\right)^{\prime}\left(\frac{\beta}{2} G_{\mu_{b}}\left(\rho_{b}\right)\right)<0$, and therefore there exists

$$
\theta_{x}^{\beta} \in\left[\frac{\beta}{2} G_{\mu_{a}}\left(\rho_{a}\right), \frac{\beta}{2} G_{\mu_{b}}\left(\rho_{b}\right)\right)
$$

such that $I_{x}^{\beta}$ is increasing on $\left[\frac{\beta}{2} G_{\mu_{a}}\left(\rho_{a}\right), \theta_{x}^{\beta}\right]$ and then decreasing. One can check that the point where $\left(I_{x}^{\beta}\right)^{\prime}$ cancels is given by $\frac{\beta}{2} Q_{\mu_{b}}\left(x-\rho_{a}\right)$. Moreover, $\left(I_{x}^{\beta}\right)^{\prime}$ is decreasing on $\left[\frac{\beta}{2} G_{\mu_{b}}\left(\rho_{b}\right), \infty\right)$ and negative at $\frac{\beta}{2} G_{\mu_{b}}\left(\rho_{b}\right)$ so it remains negative and $I_{x}^{\beta}$ is decreasing on this interval. The first claim holds true.

In Case 2, $\left(I_{x}^{\beta}\right)^{\prime}\left(\frac{\beta}{2} G_{\mu_{b}}\left(\rho_{b}\right)\right) \geq 0$, and therefore $I_{x}^{\beta}$ is increasing on $\left[\frac{\beta}{2} G_{\mu_{a}}\left(\rho_{a}\right)\right.$, $\left.\frac{\beta}{2} G_{\mu_{b}}\left(\rho_{b}\right)\right]$. But $\left(I_{x}^{\beta}\right)^{\prime}$ is nonnegative at $\frac{\beta}{2} G_{\mu_{b}}\left(\rho_{b}\right)$, decreasing on $\left[\frac{\beta}{2} G_{\mu_{b}}\left(\rho_{b}\right), \infty\right)$ and converges to $x-\rho_{a}-\rho_{b}<0$ as $\theta$ grows to $\infty$. Therefore, there exists $\theta_{x}^{\beta} \in\left(\frac{\beta}{2} G_{\mu_{b}}\left(\rho_{b}\right), \infty\right)$ such that $I_{x}^{\beta}$ is increasing on $\left(\frac{\beta}{2} G_{\mu_{b}}\left(\rho_{b}\right), \theta_{x}^{\beta}\right]$ and then decreasing. One can check that the point where $\left(I_{x}^{\beta}\right)^{\prime}$ cancels is given by $\frac{\beta}{2} \alpha_{x}$ and the second claim holds true. This concludes the proof of the uniqueness of $\theta$.

Moreover, looking carefully at the definition of $\theta_{x}^{\beta}$ in Case 1 and Case 2, one can see that it is an increasing function of $x$. Indeed, it is increasing on the intervals $\{x \in$ 
$\left.\mathbb{R} / \alpha_{x} \geq G_{\mu_{b}}\left(\rho_{b}\right)\right\}$ and $\left\{x \in \mathbb{R} / G_{\mu_{a}}\left(\rho_{a}\right) \leq \alpha_{x}<G_{\mu_{b}}\left(\rho_{b}\right)\right\}$ respectively and it is continuous at $x=G_{\mu_{b}}\left(\rho_{b}\right)$. As a consequence, for $x \neq y$ such that $\mathrm{r}\left(\mu_{a} \boxplus \mu_{b}\right) \leq x, y<\rho_{a}+\rho_{b}, \theta_{x}^{\beta} \neq \theta_{y}^{\beta}$ and therefore $\sup _{\theta \geq 0} I^{\beta}(\theta, y)>I^{\beta}\left(\theta_{x}^{\beta}, y\right)$.

We now have to deal with the case when $y=\rho_{a}+\rho_{b}$, that is to show that:

$$
\sup _{\theta \geq 0} I^{\beta}\left(\theta, \rho_{a}+\rho_{b}\right)>I^{\beta}\left(\theta_{x}^{\beta}, \rho_{a}+\rho_{b}\right)
$$

If $G_{\mu_{b}}\left(\rho_{b}\right)$ is finite, for $\theta>\frac{\beta}{2} G_{\mu_{b}}\left(\rho_{b}\right)$,

$$
I^{\beta}\left(\theta, \rho_{a}+\rho_{b}\right)=\frac{\beta}{2} \log \theta+C_{3}
$$

and therefore the supremum is infinite and (4.2) holds. Assume now that $G_{\mu_{b}}\left(\rho_{b}\right)=\infty$. As $\mu_{b} \neq \delta_{\rho_{b}}$, then, there exists $\alpha \in(0,1]$ and $M$ finite such that, for any $x \geq \rho_{b}$,

$$
G_{\mu_{b}}(x) \leq \frac{1-\alpha}{x-\rho_{b}}+M
$$

From there, we get that, for any $u>G_{\mu_{a}}\left(\rho_{a}\right) \vee \frac{2 M}{\alpha}$,

$$
u \leq \frac{1-\alpha}{K_{\mu_{b}}(u)-\rho_{b}}+M \quad \text { so that } \quad R_{\mu_{b}}(u) \leq \rho_{b}+\frac{1-\alpha}{\left(1-\frac{\alpha}{2}\right) u}-\frac{1}{u} \leq \rho_{b}-\frac{\alpha}{2 u} .
$$

Therefore, there exist $c, c^{\prime} \in \mathbb{R}$, such that for any $\theta \geq G_{\mu_{a}}\left(\rho_{a}\right) \vee \frac{2 M}{\alpha}$,

$$
I^{\beta}\left(\theta, \rho_{a}+\rho_{b}\right) \geq \theta \rho_{b}-\frac{\beta}{2} \int_{\frac{2 M}{\alpha}}^{\frac{2 \theta}{\beta}}\left(\rho_{b}-\frac{\alpha}{2 u}\right) d u+c=\frac{\beta \alpha}{4} \log \theta+c^{\prime}
$$

so that, letting $\theta$ grow to infinity, we get again that $I^{\beta}\left(\rho_{a}+\rho_{b}\right)=\infty$ and (4.2) holds. This concludes the proof of Lemma 4.2 .

\section{Large deviation lower bound}

The goal of this section is to show Proposition 2.4. A classical strategy to get a large deviation lower bound is to tilt the measure in such a way that the rare event $\left\{\lambda_{\max }^{N} \in[x-\delta, x+\delta]\right\}$ becomes typical under the tilted measure. We now check that it is possible to make such a tilt $^{1}$ :

Lemma 5.1. Under Assumptions 2.1, for any $x \in\left[\mathrm{r}\left(\mu_{a} \boxplus \mu_{b}\right), \rho_{a}+\rho_{b}\right)$ such that

$$
G_{\mu_{a} \boxplus \mu_{b}}(x) \leq \min \left(G_{\mu_{a}}\left(\rho_{a}\right), G_{\mu_{b}}\left(\rho_{b}\right)\right),
$$

for $\beta=1$ or 2 , we have

$$
\lim _{\delta \downarrow 0} \liminf _{N \rightarrow \infty} \frac{1}{N} \log m_{N}^{\beta, \theta_{x}^{\beta}}\left(\mathrm{E}_{N, \delta}^{x}\right) \geq 0,
$$

where $\mathrm{E}_{N, \delta}^{x}$ was defined in (3.6) and $\theta_{x}^{\beta}$ in Lemma 4.2.

Proof of Lemma 5.1. Let $\beta=1$ or 2 . The first remark is that, almost surely, $\left|\lambda_{\max }^{N}\right| \leq K$, where we recall that $K:=\sup _{N \geq 1}\left(\left\|A_{N}\right\|+\left\|B_{N}\right\|\right)$.

Let $\mathrm{r}\left(\mu_{a} \boxplus \mu_{b}\right) \leq x<\rho_{a}+\rho_{b}$ be fixed. If we denote by

$$
L_{x}^{\beta}(y):= \begin{cases}\sup _{\theta \geq 0} I^{\beta}(\theta, y)-I^{\beta}\left(\theta_{x}^{\beta}, y\right), & \text { if } r\left(\mu_{a} \boxplus \mu_{b}\right) \leq y \leq \rho_{a}+\rho_{b}, \\ \infty, & \text { otherwise }\end{cases}
$$

\footnotetext{
${ }^{1}$ As for Lemma 4.2, we want to mention that Lemma 5.1 holds without Assumption 4.1 , that we add to simplify the proof.
} 
we know from Proposition 3.1 that, for any $y \in \mathbb{R}$,

$$
\lim _{\delta \downarrow 0} \limsup _{N \rightarrow \infty} \frac{1}{N} \log m_{N}^{\beta, \theta_{x}^{\beta}}\left(\lambda_{\max }^{N} \in[y-\delta, y+\delta]\right) \leq-L_{x}^{\beta}(y) .
$$

Let $\delta>0$ be fixed. We denote by $F_{\delta}$ the compact set $[-K, K] \backslash(x-\delta, x+\delta)$. For any $\eta>0, y \in F_{\delta}$, we also denote by

$$
L_{x, \eta}^{\delta}(y):=\min \left(L_{x}^{\beta}(y)-\eta, \frac{1}{\eta}\right) .
$$

From (5.1), for any $y \in F_{\delta}$, there exists $\gamma_{y, \eta}>0$ such that

$$
\limsup _{N \rightarrow \infty} \frac{1}{N} \log m_{N}^{\beta, \theta_{x}^{\beta}}\left(\lambda_{\max }^{N} \in\left[y-\gamma_{y, \eta}, y+\gamma_{y, \eta}\right]\right) \leq-L_{x, \eta}^{\delta}(y) .
$$

As $F_{\delta}$ is a compact set, one can extract from the family $\left(\left[y-\gamma_{y, \eta}, y+\gamma_{y, \eta}\right]\right)_{y \in F_{\delta}}$ a finite covering $F_{\delta}=\cup_{i=1}^{r}\left[y_{i}-\gamma_{y_{i}, \eta}, y_{i}+\gamma_{y_{i}, \eta}\right]$. From there, we get that

$$
\limsup _{N \rightarrow \infty} \frac{1}{N} \log m_{N}^{\beta, \theta_{x}^{\beta}}\left(\lambda_{\max }^{N} \in F\right) \leq \max _{1 \leq i \leq r}-L_{x, \eta}^{\beta}\left(y_{i}\right) \leq-\inf _{y \in F} L_{x, \eta}^{\beta}(y) .
$$

Letting $\eta$ going to zero, we deduce that

$$
\limsup _{N \rightarrow \infty} \frac{1}{N} \log m_{N}^{\beta, \theta_{x}^{\beta}}\left(\lambda_{\max }^{N} \in F\right) \leq-\inf _{y \in F_{\delta}} L_{x}^{\beta}(y) .
$$

By Lemma 4.2, we know that $L_{x}^{\beta}$ is nonnegative and vanishes only at $x$, so that, $\inf _{y \in F_{\delta}} L_{x}^{\delta}(y)>0$. Therefore, we deduce that, for $N$ large enough,

$$
m_{N}^{\beta, \theta_{x}^{\beta}}\left(\lambda_{\max }^{N} \in[x-\delta, x+\delta]\right) \geq \frac{3}{4} .
$$

But, in virtue of Lemma 3.3, for $N$ large enough, we also have

$$
m_{N}^{\beta, \theta_{x}^{\beta}}\left(\mathrm{d}\left(\hat{\mu}_{N}, \nu_{N}^{\beta}\right) \leq N^{-1 / 4}\right) \geq \frac{3}{4}
$$

so that

and Lemma 5.1 follows.

$$
m_{N}^{\beta, \theta_{x}^{\beta}}\left(\mathrm{E}_{N, \delta}^{x}\right) \geq \frac{1}{2}
$$

From there, one can easily get the large deviation lower bound.

Proof of Proposition 2.4. As mentioned in Section 4, without loss of generality, one can assume Assumption 4.1. Let $\beta=1$ or 2 and $x \in \mathbb{R}$ be fixed. If $x>\rho_{a}+\rho_{b}$ or $x<\mathrm{r}\left(\mu_{a} \boxplus \mu_{b}\right)$, Lemma 2.2 gives that $I^{\beta}(x)=\infty$, so that the lower bound obviously holds. Moreover, as we have seen at the end of the proof of Lemma 4.2, as $\mu_{b}$ is not a Dirac mass at $\rho_{b}$, then $I^{\beta}\left(\rho_{a}+\rho_{b}\right)=\infty$ and the lower bound also holds for $x=\rho_{a}+\rho_{b}$.

Let us now assume that $\mathrm{r}\left(\mu_{a} \boxplus \mu_{b}\right) \leq x<\rho_{a}+\rho_{b}$ and let $\theta_{x}^{\beta}$ be the corresponding shift defined in Lemma 4.2. Then, with $\mathrm{E}_{N, \delta}^{x}$ defined in (3.6) and recalling that $A=A_{N}, B=$ $B_{N}$ and $H=A+U B U^{*}$, we have:

$$
\begin{array}{r}
m_{N}^{\beta}\left(\lambda_{\max }^{N} \in[x-\delta, x+\delta]\right) \geq m_{N}^{\beta}\left(\mathrm{E}_{N, \delta}^{x}\right)=\mathbb{E}_{m_{N}^{\beta}}\left(1_{\mathrm{E}_{N, \delta}^{x}} \frac{I_{N}^{\beta}\left(\theta_{x}^{\beta}, H\right)}{I_{N}^{\beta}\left(\theta_{x}^{\beta}, H\right)}\right) \\
\geq \inf _{U \in \mathrm{E}_{N, \delta}^{x}} \frac{1}{I_{N}^{\beta}\left(\theta_{x}^{\beta}, A+U B U^{*}\right)} \\
\quad \times I_{N}^{\beta}\left(\theta_{x}^{\beta}, A\right) I_{N}^{\beta}\left(\theta_{x}^{\beta}, B\right) m_{N}^{\beta, \theta_{x}^{\beta}}\left(\mathrm{E}_{N, \delta}^{x}\right)
\end{array}
$$


so that, using again Lemma 3.4, we get:

$$
\begin{aligned}
\liminf _{N \rightarrow \infty} \frac{1}{N} \log m_{N}^{\beta}\left(\lambda_{\max }^{N} \in[x-\delta, x+\delta]\right) & \geq-I^{\beta}\left(\theta_{x}^{\beta}, x\right)-g_{\theta_{x}^{\beta}}(\delta) \\
& +\liminf _{N \rightarrow \infty} \frac{1}{N} \log m_{N}^{\beta, \theta_{x}^{\beta}}\left(\mathrm{E}_{N, \delta}^{x}\right) .
\end{aligned}
$$

Letting $\delta$ going to zero and using Lemma 5.1, we get that

$$
\lim _{\delta \downarrow 0} \liminf _{N \rightarrow \infty} \frac{1}{N} m_{N}^{\beta}\left(\lambda_{\max }^{N} \in[x-\delta, x+\delta]\right) \geq-I^{\beta}\left(\theta_{x}^{\beta}, x\right) \geq-I^{\beta}(x) .
$$

This concludes the proof.

\section{Proof of the main theorem and its corollary}

Proof of Theorem 2.5. Assume that Assumption 2.1 and the condition (NoOut) are satisfied. Without loss of generality, one can add Assumption 4.1. As already stated in the proof of Lemma 5.1, almost surely, $\left|\lambda_{\max }^{N}\right| \leq K$, where we recall that $K:=$ $\sup _{N \geq 1}\left(\left\|A_{N}\right\|+\left\|B_{N}\right\|\right)$.

In particular,

$$
\limsup _{N \rightarrow \infty} \frac{1}{N} \log m_{N}^{\beta}\left(\lambda_{\max }^{N} \in[-K, K]^{c}\right)=-\infty .
$$

Using e.g. Theorem D.4(a) and Corollary D.6 in [1], it is enough to show that, for any $x \in \mathbb{R}$

$$
\begin{aligned}
\lim _{\delta \downarrow 0} \limsup _{N \rightarrow \infty} \frac{1}{N} \log m_{N}^{\beta}\left(\lambda_{\max }^{N} \in\right. & {[x-\delta, x+\delta]) } \\
& =\lim _{\delta \downarrow 0} \liminf _{N \rightarrow \infty} \frac{1}{N} \log m_{N}^{\beta}\left(\lambda_{\max }^{N} \in[x-\delta, x+\delta]\right)=-I^{\beta}(x) .
\end{aligned}
$$

The upper bound is nothing but Proposition 2.3, obtained from Proposition 3.1 for $\theta=0$ and the lower bound is given by Proposition 2.4 .

We now prove Corollary 2.6. Our goal is to show that if $A_{N}$ and $B_{N}$ have no outliers, then the condition (NoOut) is automatically satisfied. Indeed, if $A_{N}$ and $B_{N}$ have no outliers, it means that their respective largest eigenvalues converge to the edge of the support of the limiting measure, that is to say $\rho_{a}=r\left(\mu_{a}\right)$ and $\rho_{b}=r\left(\mu_{b}\right)$. Therefore, Corollary 2.6 is a direct consequence of the following lemma:

Lemma 6.1. For any probability measures $\mu$ and $\nu$ compactly supported on $\mathbb{R}$, we have

$$
G_{\mu \boxplus \nu}(\mathrm{r}(\mu \boxplus \nu)) \leq \min \left(G_{\mu}(\mathrm{r}(\mu)), G_{\nu}(\mathrm{r}(\nu))\right) .
$$

Proof. If one of the measures $\mu$ or $\nu$ is a single point mass, the additive free convolution is just a translation and we have equality. We now assume that none of them is a single point mass. In general, we know (see e.g. [6]) that there exists a function $\omega$, called the subordination function, which is analytic on $\mathbb{C}^{+}:=\{z \in \mathbb{C}, \mathfrak{I m} z>0\}$ such that, for all $z \in \mathbb{C}^{+}$,

$$
G_{\mu \boxplus \nu}(z)=G_{\mu}(\omega(z)) .
$$

This gives immediately that for any $z \in \mathbb{C}^{+}$,

$$
\mathfrak{I m} G_{\mu \boxplus \nu}(z)=-\mathfrak{I m} \omega(z) \cdot \int \frac{\mathrm{d} \mu(t)}{|t-\omega(z)|^{2}} .
$$

By [5, Theorem 2.3], as $\mu$ or $\nu$ are not a single point mass, $G_{\mu \boxplus \nu}$ can be continuously extended to $\mathbb{C}^{+} \cup \mathbb{R}$ with values in $\overline{\mathbb{C}}:=\mathbb{C} \cup\{\infty\}$. Moreover, as $\mu$ and $\nu$ are compactly 
supported, by [6, Theorem 3.3(3)], $\omega$ can also be continuously extended to $\mathbb{C}^{+} \cup \mathbb{R}$. Let $u$ be a real number in the interval $(\mathrm{r}(\mu \boxplus \nu), \infty)$. Then $\lim _{v \downarrow 0} \int \frac{\mathrm{d} \mu(t)}{|t-\omega(u+\mathrm{i} v)|^{2}}>0$ and $\lim _{v \downarrow 0} \mathfrak{I m} G_{\mu \boxplus \nu}(u+\mathrm{i} v)=\mathfrak{I m} G_{\mu \boxplus \nu}(u)=0$, so that, using (6.1), we get $\mathfrak{I m} \omega(u)=$ $\lim _{v \downarrow 0} \mathfrak{I m} G_{\mu \boxplus \nu}(u+\mathrm{i} v)=0$. Therefore, $\omega$ restricted to the interval $(\mathrm{r}(\mu \boxplus \nu), \infty)$ takes values in $\mathbb{R} \cup\{\infty\}$. Moreover, as $\omega$ is continuous and $\omega(z)$ goes to $\infty$ as $z$ goes to $\infty$, we get that $\omega((r(\mu \boxplus \nu), \infty))$ is an interval of the form $(a, \infty)$.

We now want to show by contradiction that $a \geq \mathrm{r}(\mu)$. Let us assume that $a<\mathrm{r}(\mu)$. For any $y>0$, we have

$$
-\int_{a}^{\mathrm{r}(\mu)} \mathfrak{I m} G_{\mu}(x+\mathrm{i} y) \mathrm{d} x=\int_{a}^{\mathrm{r}(\mu)} \mathrm{d} \mu(t)\left(\arctan \left(\frac{r(\mu)-t}{y}\right)-\arctan \left(\frac{a-t}{y}\right)\right) .
$$

As $y$ decreases to zero, the right hand-side goes to $\frac{\pi}{2}(\mu([a, r(\mu))+\mu((a, r(\mu)])>0$. On the other hand, for any $x \in(a, \mathrm{r}(\mu)) \subset \omega((\mathrm{r}(\mu \boxplus \nu), \infty))$, there exists $x^{\prime}>\mathrm{r}(\mu \boxplus \nu)$, such that $x=\omega\left(x^{\prime}\right)$ and $\omega$ is holomorphic from a neighborhood of $x^{\prime}$ to a neighborhood of $x$. As

$$
\lim _{\tilde{x} \rightarrow x} \mathfrak{I m} G_{\mu}(\tilde{x})=\lim _{\tilde{x}^{\prime} \rightarrow x^{\prime}} \mathfrak{I m} G_{\mu}\left(\omega\left(\tilde{x}^{\prime}\right)\right)=\mathfrak{I m} G_{\mu \boxplus \nu}\left(x^{\prime}\right)=0,
$$

by dominated convergence, we get that the left hand-side goes to zero, as $y$ decreases to zero. This leads to a contradiction and we deduce that $\omega((r(\mu \boxplus \nu), \infty)) \subset(r(\mu), \infty)$, so that

$$
\omega(r(\mu \boxplus \nu)) \geq r(\mu)
$$

As $G_{\mu}$ is decreasing on $(r(\mu), \infty)$, this gives

$$
G_{\mu \boxplus \nu}(\mathrm{r}(\mu \boxplus \nu))=G_{\mu}(\omega(\mathrm{r}(\mu \boxplus \nu))) \leq G_{\mu}(\mathrm{r}(\mu)) .
$$

As $\mu$ and $\nu$ play symmetric roles, this concludes the proof of Lemma 6.1.

\section{A Study of the deformed model (2.4)}

\section{A.1 Large deviations for the smallest eigenvalue of $H_{N}$}

In order to study the deviations of the largest eigenvalue of the deformed model below its expected value, we will need a counterpart of Theorem 2.5 for the smallest eigenvalue of $H_{N}$. We first state the counterpart of the condition (NoOut). For any compactly supported probability measure $\mu$, we denote by $(\mu)$ the left edge of the support of $\mu$. One can extend the definitions of $G_{\mu}, K_{\mu}, R_{\mu}$ and $Q_{\mu}$ given in Section 2: for any $\lambda<\mathrm{I}(\mu)$, $G_{\mu}(\lambda):=\int \frac{1}{\lambda-y} \mu(\mathrm{d} y) ; G_{\mu}$ is decreasing from $(-\infty, \mathrm{I}(\mu))$ into $\left(G_{\mu}(\mathrm{I}(\mu)), 0\right)$ so we denote again by $K_{\mu}$ its inverse. For any $z \in\left(G_{\mu}(\mathrm{I}(\mu)), 0\right)$ we set $R_{\mu}(z):=K_{\mu}(z)-\frac{1}{z}$, which is increasing with inverse $Q_{\mu}$. We then introduce the following assumption:

(NoDown) The smallest eigenvalues $\lambda_{N}^{\left(A_{N}\right)}$ and $\lambda_{N}^{\left(B_{N}\right)}$ converge as $N$ grows to infinity to $\ell_{a}$ and $\ell_{b}$ respectively and $G_{\mu_{a} \boxplus \mu_{b}}\left(I\left(\mu_{a} \boxplus \mu_{b}\right)\right) \geq \max \left(G_{\mu_{a}}\left(\ell_{a}\right), G_{\mu_{b}}\left(\ell_{b}\right)\right)$.

As in Lemma 6.1, one can check that this condition is satisfied if $A_{N}$ and $B_{N}$ have no outliers, this time in the sense that $\ell_{a}=\mathrm{I}\left(\mu_{a}\right)$ and $\ell_{b}=\mathrm{I}\left(\mu_{b}\right)$. We now extend the definition of the rate function $I^{\beta}$ introduced in (2.1). For $\beta=1$ or $2, \theta \leq 0, \mu$ a compactly supported probability measure and $\ell \leq \mathrm{I}(\mu)$, we define:

$$
J_{\mu}^{\beta}(\theta, \ell):= \begin{cases}\frac{\beta}{2} \int_{0}^{\frac{2 \theta}{\beta}} R_{\mu}(u) \mathrm{d} u, & \text { if } G_{\mu}(\ell) \leq \frac{2 \theta}{\beta} \leq 0, \\ \theta \ell-\frac{\beta}{2} \log (-\theta)-\frac{\beta}{2} \int \log (y-\ell) \mu(\mathrm{d} y)+\frac{\beta}{2}\left(\log \frac{\beta}{2}-1\right), & \text { if } \frac{2 \theta}{\beta}<G_{\mu}(\ell) .\end{cases}
$$

For any $\theta \leq 0$ and $x \leq \mathrm{I}\left(\mu_{a} \boxplus \mu_{b}\right)$, we denote by

$$
I^{\beta}(\theta, x):=J_{\mu_{a} \boxplus \mu_{b}}^{\beta}(\theta, x)-J_{\mu_{a}}^{\beta}\left(\theta, \ell_{a}\right)-J_{\mu_{b}}^{\beta}\left(\theta, \ell_{b}\right),
$$


and

$$
I_{\min }^{\beta}(x):= \begin{cases}\sup _{\theta \leq 0} I^{\beta}(\theta, x), & \text { if } x \leq \mathrm{I}\left(\mu_{a} \boxplus \mu_{b}\right), \\ \infty, & \text { otherwise. }\end{cases}
$$

Applying Theorem 2.5 to $-A_{N}$ and $-B_{N}$, one can get a large deviation principle for the smallest eigenvalue $\lambda_{\min }^{N}$ of $H_{N}$ :

Corollary A.1. Under the assumptions ( $H_{\mathrm{bulk}}$ ) and (NoDown), for $\beta=1$ or 2 , the law of $\lambda_{\min }^{N}$ under $m_{N}^{\beta}$ satisfies a large deviation principle in the scale $N$ with good rate function $I_{\min }^{\beta}$.

\section{A.2 Asymptotic independence of the deviations of $\lambda_{\min }^{N}$ and $\lambda_{\max }^{N}$}

Before going to the study of the deformed model itself, we will need the following proposition:

Proposition A.2. Let $\left(M_{N}\right)_{N \geq 1}$ be a sequence of deterministic matrices such that $M:=$ $\sup _{N>1}\left\|M_{N}\right\|<\infty$, and, as $N$ goes to infinity, $\lambda_{1}^{\left(M_{N}\right)}$ and $\lambda_{N}^{\left(M_{N}\right)}$ converge respectively to $\rho$ and $\ell$ and $\hat{\mu}_{M_{N}}$ converges weakly to $\mu$. Let $e_{1}$ and $e_{2}$ be two random vectors uniformly distributed on the unit sphere of $\mathbb{R}^{N}$ if $\beta=1$ (respectively of $\mathbb{C}^{N}$ if $\beta=2$ ), orthogonal to each other. Let $\theta \geq 0$ and $\theta^{\prime} \leq 0$ be fixed. Then

$$
\lim _{N \rightarrow \infty} \frac{1}{N} \log \frac{\mathbb{E}\left(\mathrm{e}^{N \theta\left\langle e_{1}, M_{N} e_{1}\right\rangle+N \theta^{\prime}\left\langle e_{2}, M_{N} e_{2}\right\rangle}\right)}{I_{N}\left(\theta, M_{N}\right) I_{N}\left(\theta^{\prime}, M_{N}\right)}=0 .
$$

In other words, when $\theta$ and $\theta^{\prime}$ are of opposite sign, the rank two spherical integral asymptotically factorizes in the scale $\mathrm{e}^{N}$. As an immediate corollary, we find that the large deviations of $\lambda_{\min }$ and $\lambda_{\max }$ are asymptotically independent.

Corollary A.3. Under the assumptions ( $\left.H_{\mathrm{bulk}}\right)$, (NoDown) and (NoOut), for $\beta=1$ or 2 , the law of $\left(\lambda_{\min }^{N}, \lambda_{\max }^{N}\right)$ under $m_{N}^{\beta}$ satisfies a large deviation principle in the scale $N$ and with good rate function $I_{\min }^{\beta}(x)+I^{\beta}(y)$.

Proof. The proof is to tilt the measure by the rank two spherical integral of Proposition A.2 which implies that for $\theta>0$ and $\theta^{\prime}<0$

$$
\begin{aligned}
& m_{N}\left(\left|\lambda_{\text {min }}^{N}-x\right|+\left|\lambda_{\text {max }}^{N}-y\right| \leq \delta\right) \\
\leq & e^{-N\left(J_{\mu_{A} \boxplus \mu_{B}}^{\beta}\left(\theta^{\prime}, x\right)+J_{\mu_{A} \boxplus \mu_{B}}^{\beta}(\theta, y)+o(1)\right)} \mathbb{E}_{U}\left[\mathbb{E}_{e}\left(\mathrm{e}^{N \theta\left\langle e_{1},\left(A+U B U^{*}\right) e_{1}\right\rangle+N \theta^{\prime}\left\langle e_{2},\left(A+U B U^{*}\right) e_{2}\right\rangle}\right)\right]
\end{aligned}
$$

Now, since the law of $\left(e_{1}, e_{2}\right)$ and $\left(U e_{1}, U e_{2}\right)$ are independent and equidistributed, we deduce the upper bound as before. The proof of the lower bound is the same since for any $(x, y)$ we find a unique couple $\left(\theta_{x}^{\prime}, \theta_{y}\right)$ which optimizes the rate function.

Moreover, it is easy to deduce the following corollary, which is the extension of Proposition 2.3 to $\theta<0$ :

Corollary A.4. Under Assumption 2.1, for $\beta=1$ or 2 , for any $\theta<0$, for any $x<r\left(\mu_{a} \boxplus \mu_{b}\right)$,

$$
\lim _{\delta \downarrow 0} \limsup _{N \rightarrow+\infty} \frac{1}{N} \log m_{N}^{\beta, \theta}\left(\lambda_{\max }^{N} \in[x-\delta, x+\delta]\right)=-\infty
$$

and for any $x \geq r\left(\mu_{a} \boxplus \mu_{b}\right)$,

$$
\lim _{\delta \downarrow 0} \limsup _{N \rightarrow+\infty} \frac{1}{N} \log m_{N}^{\beta, \theta}\left(\lambda_{\max }^{N} \in[x-\delta, x+\delta]\right) \leq-I^{\beta}(x) .
$$


With Proposition A.2 in hand, the proof of Corollary A.4 follows the same lines as the proof of Proposition 2.3. We do not detail it and go directly to the proof of Proposition A.2. Note that this kind of factorization property has been already shown for $\theta$ and $\theta^{\prime}$ not too far from zero, we refer the reader to [20, Theorem 7] or [15]. Our goal here is to extend this result to any pair of $\left(\theta, \theta^{\prime}\right)$ of opposite sign.

Proof of Proposition A.2. For the sake of simplicity, we will stick to the case $\beta=1$. Let $g$ and $g^{\prime}$ be two independent standard Gaussian vectors in $\mathbb{R}^{N}$. If we denote by $\|\cdot\|_{2}$ the Euclidean norm and set

$$
e_{1}:=\frac{g}{\|g\|_{2}}, h:=g^{\prime}-\frac{\left\langle g, g^{\prime}\right\rangle}{\|g\|_{2}^{2}} g \text { and } e_{2}:=\frac{h}{\|h\|_{2}},
$$

then it is well known that $\left(e_{1}, e_{2}\right)$ are two random vectors uniform on the unit sphere in $\mathbb{R}^{N}$, orthogonal to each other. Moreover, $\left(e_{1}, e_{2}\right)$ is independent from $\left(\|g\|_{2},\left\|g^{\prime}\right\|_{2},\left\langle g, g^{\prime}\right\rangle\right)$. Indeed, one can use the following system of coordinates: $r:=\|g\|_{2}, \gamma_{1}, \ldots, \gamma_{N-1}$ are the polar coordinates of $g, r^{\prime}:=\left\|g^{\prime}\right\|_{2}, \eta$ is the angle between $g$ and $g$, and $\gamma_{1}^{\prime}, \ldots, \gamma_{N-2}^{\prime}$ are the angles needed to spot $g^{\prime}$ on the cone of angle $\eta$ around $g$. One can check that the Gaussian measure decomposes as a product measure in these coordinates, $\left(\|g\|_{2},\left\|g^{\prime}\right\|_{2},\left\langle g, g^{\prime}\right\rangle\right)$ is a function of $r, r^{\prime}, \eta$ whereas $\left(e_{1}, e_{2}\right)$ is a function of the $\gamma^{\prime}$ s and $\gamma^{\prime}$ s. In particular, for any $\varepsilon$, if we let

$$
A_{N}^{\varepsilon}:=\left\{\left|\left\langle g, g^{\prime}\right\rangle\right| \leq \varepsilon\|g\|_{2}\left\|g^{\prime}\right\|_{2}\right\}
$$

then $A_{N}^{\varepsilon}$ is independent of $\left(e_{1}, e_{2}\right)$. Moreover, on $A_{N}^{\varepsilon}$, we have $\|h\|_{2}^{2} \geq\left\|g^{\prime}\right\|_{2}^{2}\left(1-\varepsilon^{2}\right)$ so that, for $\varepsilon<1 / 2$,

$$
\left|\theta\left\langle e_{1}, M_{N} e_{1}\right\rangle+\theta^{\prime}\left\langle e_{2}, M_{N} e_{2}\right\rangle-\theta \frac{1}{\|g\|_{2}}\left\langle g, M_{N} g\right\rangle-\theta^{\prime} \frac{1}{\left\|g^{\prime}\right\|_{2}}\left\langle g^{\prime}, M_{N} g^{\prime}\right\rangle\right| \leq 4 M \varepsilon
$$

and

$$
\begin{aligned}
\mathbb{E}\left(\mathrm{e}^{N \theta\left\langle e_{1}, M_{N} e_{1}\right\rangle+N \theta^{\prime}\left\langle e_{2}, M_{N} e_{2}\right\rangle}\right) & =\frac{1}{\mathrm{P}\left(A_{N}^{\varepsilon}\right)} \mathbb{E}\left(1_{A_{N}^{\varepsilon}} \mathrm{e}^{N \theta\left\langle e_{1}, M_{N} e_{1}\right\rangle+N \theta^{\prime}\left\langle e_{2}, M_{N} e_{2}\right\rangle}\right) \\
& \leq \frac{\mathrm{e}^{4 N M \varepsilon}}{\mathrm{P}\left(A_{N}^{\varepsilon}\right)} I_{N}\left(\theta, M_{N}\right) I_{N}\left(\theta^{\prime}, M_{N}\right) .
\end{aligned}
$$

Because of the law of large numbers, for any $\varepsilon>0, \mathbb{P}\left(A_{N}^{\varepsilon}\right)$ converges to 1 as $N$ goes to infinity. Hence, letting $N$ go to infinity and then $\varepsilon$ going to zero, we get the upper bound in (A.2).

We now prove the lower bound. If $O$ is an orthogonal matrix, the law of $\left(O e_{1}, O e_{2}\right)$ is the same as the law of $\left(e_{1}, e_{2}\right)$ so that we can assume without loss of generality that $M_{N}$ is real diagonal, with eigenvalues that we denote by $\lambda_{1}^{N} \geq \lambda_{2}^{N} \geq \ldots \geq \lambda_{N}^{N}$. We refer the reader to Proposition 16 and Lemmas 18 to 21 in [20], in particular the proof of Lemma 19. We recall that $T$ is the rate function for the large deviations of $\frac{\sum_{i=1}^{N} \lambda_{i}^{N} g_{i}^{2}}{\sum_{i=1}^{N} g_{i}^{2}}$. Let $\alpha_{1}^{*}$ be such that

$$
\theta \alpha_{1}^{*}-T\left(\alpha_{1}^{*}\right)=\sup _{\alpha}(\theta \alpha-T(\alpha))
$$

As $\theta \geq 0$, one can check that $\alpha_{\min } \leq \alpha_{1}^{*} \leq \rho$. If $\alpha_{1}^{*} \in\left[\alpha_{\min }, \alpha_{\max }\right]$, we set $x_{1}:=0$, whereas if $\alpha_{1}^{*} \in\left(\alpha_{\max }, \rho\right)$, we set $x_{1}:=\left(\rho-\alpha_{1}^{*}\right)\left(\left(\rho-\alpha_{1}^{*}\right) H_{\max }-1\right)$. Similarly, let $\alpha_{2}^{*}$ be such that

$$
\theta^{\prime} \alpha_{2}^{*}-T\left(\alpha_{2}^{*}\right)=\sup _{\alpha}\left(\theta^{\prime} \alpha-T(\alpha)\right)
$$


As $\theta^{\prime}<0$, one can check that $\ell \leq \alpha_{2}^{*} \leq \alpha_{\max }$. If $\alpha_{2}^{*} \in\left[\alpha_{\min }, \alpha_{\max }\right]$, we set $x_{2}:=0$, whereas if $\alpha_{2}^{*} \in\left(\ell, \alpha_{\min }\right)$, we set $x_{2}:=\left(\ell-\alpha_{1}^{*}\right)\left(\left(\ell-\alpha_{1}^{*}\right) H_{\min }-1\right)$. We now define, for any $\delta>0$,

$$
\begin{aligned}
B_{\alpha_{1}^{*}, x_{1}, \alpha_{2}^{*}, x_{2}}^{\delta}:=\left\{\left|\left(\lambda_{1}^{N}-\alpha_{1}^{*}\right) \frac{g_{1}^{2}}{N}+x_{1}\right| \leq \delta,\left|\frac{1}{N} \sum_{i=2}^{N}\left(\lambda_{i}^{N}-\alpha_{1}^{*}\right) g_{i}^{2}-x_{1}\right| \leq \delta,\right. \\
\left.\quad\left|\left(\lambda_{N}^{N}-\alpha_{2}^{*}\right) \frac{\left(g_{N}^{\prime}\right)^{2}}{N}+x_{2}\right| \leq \delta,\left|\frac{1}{N} \sum_{i=1}^{N-1}\left(\lambda_{i}^{N}-\alpha_{2}^{*}\right)\left(g_{i}^{\prime}\right)^{2}-x_{2}\right| \leq \delta\right\}, \\
C:=\left\{\forall i \geq 2, g_{i}^{2} \leq N^{1 / 4}, \forall i \leq N-1,\left(g_{i}^{\prime}\right)^{2} \leq N^{1 / 4}\right\}, \\
E_{\delta}:=\left\{\|g\|^{2} \geq \sqrt{\delta} N,\left\|g^{\prime}\right\|^{2} \geq \sqrt{\delta} N\right\}
\end{aligned}
$$

We have

$$
\begin{gathered}
\mathbb{E}\left(\mathrm{e}^{N \theta\left\langle e_{1}, M_{N} e_{1}\right\rangle+N \theta^{\prime}\left\langle e_{2}, M_{N} e_{2}\right\rangle}\right) \geq \mathbb{E}\left(\mathbf{1}_{A_{N}^{\varepsilon} \cap B_{\alpha_{1}^{*}, x_{1}, \alpha_{2}^{*}, x_{2}}^{\delta} \cap C \cap E_{\delta}} \mathrm{e}^{N \theta\left\langle e_{1}, M_{N} e_{1}\right\rangle+N \theta^{\prime}\left\langle e_{2}, M_{N} e_{2}\right\rangle}\right) \\
\quad \geq E\left(\mathbf{1}_{A_{N}^{\varepsilon} \cap B_{\alpha_{1}^{*}, x_{1}, \alpha_{2}^{*}, x_{2}}^{\delta} \cap C \cap E_{\delta}} \mathrm{e}^{\theta \frac{1}{\|g\|_{2}}\left\langle g, M_{N} g\right\rangle-\theta^{\prime} \frac{1}{\left\|g^{\prime}\right\|_{2}}\left\langle g^{\prime}, M_{N} g^{\prime}\right\rangle}\right) \mathrm{e}^{-4 N M \varepsilon} \\
\quad \geq \mathbb{P}\left(A_{N}^{\varepsilon} \cap B_{\alpha_{1}^{*}, x_{1}, \alpha_{2}^{*}, x_{2}}^{\delta} \cap C \cap E_{\delta}\right) \mathrm{e}^{-4 N M \varepsilon} \mathrm{e}^{N \theta \alpha_{1}^{*}+N \theta^{\prime} \alpha_{2}^{*}-2 N\left(\theta+\theta^{\prime}\right) \sqrt{\delta}}
\end{gathered}
$$

Now, if $\sigma_{1}, \ldots, \sigma_{N}$ are $N$ independent Rademacher random variables, independent of $g$ and $g^{\prime}$, then $\left\langle g, g^{\prime}\right\rangle$ and $\sum_{i=1}^{N} \sigma_{i} g_{i} g_{i}^{\prime}$ have the same law. Therefore, since the sets $B_{\alpha_{1}^{*}, x_{1}, \alpha_{2}^{*}, x_{2}}^{\delta}, C$ and $E_{\delta}$ are independent of the sign of the $g_{i}{ }^{\prime}$,

$\mathbb{P}\left(A_{N}^{\varepsilon} \mid B_{\alpha_{1}^{*}, x_{1}, \alpha_{2}^{*}, x_{2}}^{\delta} \cap C \cap E_{\delta}\right)=\mathbb{P}\left(\mathbb{P}\left(\left|\sum_{i=1}^{N} \sigma_{i} g_{i} g_{i}^{\prime}\right| \leq \varepsilon\|g\|_{2}\left\|g^{\prime}\right\|_{2} \mid\left(g, g^{\prime}\right)\right) \mid B_{\alpha_{1}^{*}, x_{1}, \alpha_{2}^{*}, x_{2}}^{\delta} \cap C \cap E_{\delta}\right)$

where the second expectation holds on the $\sigma^{\prime}$ s only. Using the concentration properties of the Rademacher random variables (or the Azuma Hoeffding inequality), one gets that

$$
\mathbb{P}\left(\left|\sum_{i=1}^{N} \sigma_{i} g_{i} g_{i}^{\prime}\right| \geq \varepsilon\|g\|_{2}\left\|g^{\prime}\right\|_{2} \mid\left(g, g^{\prime}\right)\right) \leq \mathrm{e}^{-\frac{\varepsilon^{2}\|g\|_{2}^{2}\left\|g^{\prime}\right\|_{2}^{2}}{\sum_{i=1}^{N} g_{i}^{2}\left(g_{i}^{\prime}\right)^{2}}}
$$

On $B_{\alpha_{1}^{*}, x_{1}, \alpha_{2}^{*}, x_{2}}^{\delta} \cap C \cap E_{\delta}$, the right hand side is bounded above by $\mathrm{e}^{-4 \sqrt{N} \varepsilon^{2} \delta}$, so that we can conclude that, for any $\varepsilon, \delta>0, \mathbb{P}\left(A_{N}^{\varepsilon} \mid B_{\alpha_{1}^{*}, x_{1}, \alpha_{2}^{*}, x_{2}}^{\delta} \cap C \cap E_{\delta}\right)$ converges to one as $N$ goes to infinity.

Furthermore, we have that

$$
\mathbb{P}\left(B_{\alpha_{1}^{*}, x_{1}, \alpha_{2}^{*}, x_{2}}^{\delta} \cap C \cap E_{\delta}\right) \geq \mathbb{P}\left(B_{\alpha_{1}^{*}, x_{1}, \alpha_{2}^{*}, x_{2}}^{\delta} \cap C\right)-\mathbb{P}\left(E_{\delta}^{c}\right) .
$$

Since it is well known that

$$
\liminf _{N \rightarrow \infty} \frac{1}{N} \log \mathbb{P}\left(E_{\delta}^{c}\right) \leq-2(\sqrt{\delta}-1-\log (\sqrt{\delta}))
$$

where the above right hand side goes to $-\infty$ as $\delta$ goes to zero, we only need to estimate the first term in the right hand side of (A.6) for small enough $\delta$. Now

$$
\mathbb{P}\left(B_{\alpha_{1}^{*}, x_{1}, \alpha_{2}^{*}, x_{2}}^{\delta} \cap C\right) \geq \mathbb{P}\left(B_{\alpha_{1}^{*}, x_{1}, \alpha_{2}^{*}, x_{2}}^{\delta} \mid C\right) \mathbb{P}(C),
$$

where the last term goes to one as $N$ goes to infinity. The last thing to check is that

$$
\lim _{\delta \downarrow 0} \liminf _{N \rightarrow \infty} \frac{1}{N} \log \mathbb{P}\left(B_{\alpha_{1}^{*}, x_{1}, \alpha_{2}^{*}, x_{2}}^{\delta} \mid C\right) \geq-T\left(\alpha_{1}^{*}\right)-T\left(\alpha_{2}^{*}\right) .
$$


Indeed, going back to the proofs of Lemmas 18 and 19 in [20] (see also [11]), one can check that if $\alpha_{1}^{*} \in\left[\alpha_{\min }, \alpha_{\max }\right]$,

$$
\lim _{\delta \downarrow 0} \liminf _{N \rightarrow \infty} \frac{1}{N} \log \mathbb{P}\left(\left|\frac{1}{N} \sum_{i=2}^{N}\left(\lambda_{i}^{N}-\alpha_{1}^{*}\right) g_{i}^{2}\right| \leq \delta \mid C\right) \geq-L^{\alpha_{1}^{*}}(0) .
$$

The proof is the same except that in the computation of the log-Laplace the integral will go from $-N^{1 / 4}$ to $N^{1 / 4}$ instead of running on $\mathbb{R}$ and this will not change the limit. Similarly, if $\alpha_{1}^{*}>\alpha_{\max }$,

$$
\lim _{\delta \downarrow 0} \liminf _{N \rightarrow \infty} \frac{1}{N} \log \mathbb{P}\left(\left|\left(\lambda_{1}^{N}-\alpha_{1}^{*}\right) \frac{g_{1}^{2}}{N}+x_{1}\right| \leq \delta\right) \geq-\frac{x_{1}}{2\left(\rho-\alpha_{1}^{*}\right)},
$$

and

$$
\lim _{\delta \downarrow 0} \liminf _{N \rightarrow \infty} \frac{1}{N} \log \mathbb{P}\left(\left|\frac{1}{N} \sum_{i=2}^{N}\left(\lambda_{i}^{N}-\alpha_{1}^{*}\right) g_{i}^{2}-x_{1}\right| \leq \delta \mid C\right) \geq-L^{\alpha_{1}^{*}}\left(x_{1}\right) .
$$

Putting everything together in (A.5), taking the limit as $N$ then $\delta$ and $\varepsilon$ go to zero, we get the factorization property.

\section{A.3 Large deviations for the largest eigenvalue in the deformed model}

For the sake of simplicity, when treating the deformed model, we will stick to the case $\beta=1$. For any $x>\operatorname{r}\left(\mu_{a} \boxplus \mu_{b}\right)$, we denote by $\mu_{x}$ the measure defined as follows: for any bounded measurable function $f$,

$$
\int f(\lambda) \mu_{x}(\mathrm{~d} \lambda)=\int f\left(\frac{1}{x-\lambda}\right) \mu_{a} \boxplus \mu_{b}(\mathrm{~d} \lambda) .
$$

In particular, for any $x>\mathrm{r}\left(\mu_{a} \boxplus \mu_{b}\right), \int \lambda \mu_{x}(\mathrm{~d} \lambda)=G_{\mu_{a} \boxplus \mu_{b}}(x)$.

For any $x>\rho \geq \mathrm{r}\left(\mu_{a} \boxplus \mu_{b}\right)$ and $\ell \leq \mathrm{I}\left(\mu_{a} \boxplus \mu_{b}\right)$ we define

$$
\alpha_{x,+}(\rho):= \begin{cases}\frac{G_{\mu_{a} \boxplus \mu_{b}}(\rho)}{1+(x-\rho) G_{\mu_{a} \boxplus \mu_{b}}(\rho)}, & \text { if } G_{\mu_{a} \boxplus \mu_{b}}(\rho)<\infty, \\ \infty, & \text { otherwise }\end{cases}
$$

and

$$
\alpha_{x,-}(\ell):= \begin{cases}\frac{G_{\mu_{a} \boxplus \mu_{b}}(\ell)}{1+(x-\ell) G_{\mu_{a} \boxplus \mu_{b}}(\ell)}, & \text { if } G_{\mu_{a} \boxplus \mu_{b}}(\ell)>-\infty, \\ -\infty, & \text { otherwise. }\end{cases}
$$

For $\alpha \in\left(\frac{1}{x-\ell}, \frac{1}{x-\rho}\right)$ and $\kappa \notin\left(\frac{1}{x-\ell}, \frac{1}{x-\rho}\right)$, we set

$$
h_{\alpha, x}(\kappa):=\frac{1}{2} \int \log \left(\frac{\kappa-\lambda}{\kappa-\alpha}\right) \mu_{x}(\mathrm{~d} \lambda) .
$$

and we also extend it to

$$
h_{\alpha, x}\left(\frac{1}{x-\rho}\right):=\lim _{y \downarrow \rho} h_{\alpha, x}\left(\frac{1}{x-y}\right) \quad \text { and } \quad h_{\alpha, x}\left(\frac{1}{x-\ell}\right):=\lim _{y \uparrow \ell} h_{\alpha, x}\left(\frac{1}{x-y}\right)
$$

We set, for $x>\rho \geq \mathrm{r}\left(\mu_{a} \boxplus \mu_{b}\right)$ and $\ell \leq \mathrm{I}\left(\mu_{a} \boxplus \mu_{b}\right)$,

$$
T_{x, \rho}^{+}(\alpha):= \begin{cases}h_{\alpha, x}\left(K_{\mu_{x}}\left(Q_{\mu_{x}}(\alpha)\right)\right), & \text { if } \alpha \in\left[G_{\mu_{a} \boxplus \mu_{b}}(x), \alpha_{x,+}(\rho)\right], \\ h_{\alpha, x}\left(\frac{1}{x-\rho}\right), & \text { if } \alpha \in\left(\alpha_{x,+}(\rho), \frac{1}{x-\rho}\right), \\ \infty, & \text { if } \alpha>\frac{1}{x-\rho}\end{cases}
$$


and

$$
T_{x, \ell}^{-}(\alpha):= \begin{cases}h_{\alpha, x}\left(K_{\mu_{x}}\left(Q_{\mu_{x}}(\alpha)\right)\right), & \text { if } \alpha \in\left[\alpha_{x,-}(\ell), G_{\mu_{a} \boxplus \mu_{b}}(x)\right], \\ h_{\alpha, x}\left(\frac{1}{x-\ell}\right), & \text { if } \alpha \in\left(\frac{1}{x-\ell}, \alpha_{x,-}(\ell)\right) \\ \infty, & \text { if } \alpha<\frac{1}{x-\ell} .\end{cases}
$$

The quantities above can easily be extended to the case $x=\rho>\mathrm{r}\left(\mu_{a} \boxplus \mu_{b}\right)$ (only the first line of (A.7) will be relevant). For $x=\rho=\mathrm{r}\left(\mu_{a} \boxplus \mu_{b}\right)$, we set

$$
T_{x, x}^{+}(\alpha):=0 \quad \text { if } \alpha \geq G_{\mu_{a} \boxplus \mu_{b}}(x)
$$

and

$$
T_{x, \ell}^{-}(\alpha):=\infty \quad \text { if } \alpha<G_{\mu_{a} \boxplus \mu_{b}}(x)
$$

For $\gamma:=\left(\gamma_{1}, \ldots, \gamma_{p}\right)$ a $p$-uplet of nonnegative real numbers, we now define,

$$
L_{\gamma}^{(0)}(y):=I^{1}(y), \quad \text { if } y \geq r\left(\mu_{a} \boxplus \mu_{b}\right)
$$

and, for any $1 \leq i \leq p$,

$$
L_{\gamma}^{(i)}(x):= \begin{cases}\inf _{y \leq \mathrm{I}\left(\mu_{a} \boxplus \mu_{b}\right)}\left\{T_{x, y}^{-}\left(\frac{1}{\gamma_{i}}\right)+I_{\min }^{1}(y)\right\}, & \text { if } \mathrm{r}\left(\mu_{a} \boxplus \mu_{b}\right) \leq x<K_{\mu_{a} \boxplus \mu_{b}}\left(\frac{1}{\gamma_{i}}\right), \\ \inf _{\mathrm{r}\left(\mu_{a} \boxplus \mu_{b}\right) \leq y \leq x}\left\{T_{x, y}^{+}\left(\frac{1}{\gamma_{i}}\right)+L_{\gamma}^{(i-1)}(y)\right\}, & \text { if } x \geq K_{\mu_{a} \boxplus \mu_{b}}\left(\frac{1}{\gamma_{i}}\right), \\ \infty, & \text { if } x<\mathrm{r}\left(\mu_{a} \boxplus \mu_{b}\right),\end{cases}
$$

with the convention that

$$
K_{\mu_{a} \boxplus \mu_{b}}\left(\frac{1}{\gamma_{i}}\right)=\mathrm{r}\left(\mu_{a} \boxplus \mu_{b}\right) \quad \text { if } G_{\mu_{a} \boxplus \mu_{b}}\left(r\left(\mu_{a} \boxplus \mu_{b}\right)\right) \leq \frac{1}{\gamma_{i}} .
$$

Note that this rate function should not depend on the ordering of the $\gamma_{i}$ 's, which is far from obvious on the formula above.

We can now state our main result. We recall that $\left(U_{1}^{(1)}, \ldots, U_{1}^{(p)}\right)$ are independent random vectors uniformly distributed on the unit sphere. To simplify the notations, they can be viewed as respective first column vectors of $p$ independent matrices distributed according to $m_{N}^{1}$.

Theorem A.5. Under the assumptions ( $H_{\text {bulk }}$ ), (NoOut) and (NoDown), for any $p \in \mathbb{N}^{*}$ and any $\gamma \in\left(\mathbb{R}_{+}\right)^{p}$, the law of the largest eigenvalue $\widetilde{\lambda_{\max }^{N}}$ of the matrix $X_{N}:=A_{N}+$ $U B_{N} U^{*}+\sum_{i=1}^{p} \gamma_{i} U_{1}^{(i)}\left(U_{1}^{(i)}\right)^{*}$, defined in (2.4), under $\left(m_{N}^{1}\right)^{\otimes(p+1)}$ satisfies a large deviation principle in the scale $N$ with good rate function $L_{\gamma}^{(p)}$.

Before proving Theorem A.5, we need to state a variant of Proposition 16 in [20]. We denote by $P$ the standard Gaussian measure on $\mathbb{R}$ and we assume that $\left(g_{1}, \ldots, g_{N}\right)$ follows the law $P^{\otimes N}$. For any $N$-tuple of real numbers $\lambda:=\left(\lambda_{1}, \ldots, \lambda_{N}\right)$ and $x \notin\left\{\lambda_{1}, \ldots, \lambda_{N}\right\}$, we denote by $v_{N, \lambda}(x):=\frac{\sum_{i=1}^{N} \frac{1}{x-\lambda_{i}} g_{i}^{2}}{\sum_{i=1}^{N} g_{i}^{2}}$.

Proposition A.6. Let $\left(\lambda_{i}^{N}\right)_{N \in \mathbb{N}^{*}, 1 \leq i \leq N}$ be a triangular array of real numbers such that $\frac{1}{N} \sum_{i=1}^{N} \delta_{\lambda_{i}^{N}}$ converges to $\mu_{a} \boxplus \mu_{b}$ as $N$ grows to $\infty$. We denote by $\lambda^{N}:=\left(\lambda_{1}^{N}, \ldots, \lambda_{N}^{N}\right)$. Assume that $\max _{i=1}^{N} \lambda_{i}^{N}$ converges, as $N$ grows to $\infty$, to $\rho \geq \mathrm{r}\left(\mu_{a} \boxplus \mu_{b}\right)$. Let $x$ be a real number such that, for $N$ large enough, $x>\max _{i=1}^{N} \lambda_{i}^{N}$. Then, for any $\alpha \in \mathbb{R}$ such that $\alpha \geq G_{\mu_{a} \boxplus \mu_{b}}(x)$, we have

$$
\begin{aligned}
\lim _{\delta \downarrow 0} \limsup _{N \rightarrow \infty} \frac{1}{N} \log P^{\otimes N}\left(v_{N, \lambda^{N}}(x) \in[\alpha-\delta, \alpha+\delta]\right) \\
\quad=\lim _{\delta \downarrow 0} \liminf _{N \rightarrow \infty} \frac{1}{N} \log P^{\otimes N}\left(v_{N, \lambda^{N}}(x) \in[\alpha-\delta, \alpha+\delta]\right)=-T_{x, \rho}^{+}(\alpha) .
\end{aligned}
$$


Assume that $\min _{i=1}^{N} \lambda_{i}^{N}$ converges, as $N$ grows to $\infty$, to $\ell \leq \mathrm{I}\left(\mu_{a} \boxplus \mu_{b}\right)$. Then, for any $\alpha \in \mathbb{R}$ such that $\alpha<G_{\mu_{a} \boxplus \mu_{b}}(x)$, we have

$$
\begin{aligned}
\lim _{\delta \downarrow 0} \limsup _{N \rightarrow \infty} \frac{1}{N} & \log P^{\otimes N}\left(v_{N, \lambda^{N}}(x) \in[\alpha-\delta, \alpha+\delta]\right) \\
\quad & =\lim _{\delta \downarrow 0} \liminf _{N \rightarrow \infty} \frac{1}{N} \log P^{\otimes N}\left(v_{N, \lambda^{N}}(x) \in[\alpha-\delta, \alpha+\delta]\right)=-T_{x, \ell}^{-}(\alpha) .
\end{aligned}
$$

We will not give a full proof of Proposition A.6. This follows from an adaptation of Lemma 18 and Proposition 16 in [20]. In Lemma 18 in particular, one can check that the deviations above the mean (which is $G_{\mu_{a} \boxplus \mu_{b}}(x)$ in the present case) may involve not only the limiting empirical distribution but also the limit as $N$ grows to $\infty$ of the largest particle (denoted by $\max _{i=1}^{N} \gamma_{i}$ there and equal to $\frac{1}{x-\max _{i=1}^{N} \lambda_{i}^{N}}$ in the present case), whereas the deviations below the mean may depend on the limiting smallest particle, equal to $\frac{1}{\min _{i=1}^{N} \lambda_{i}^{N}-x}$ here.

The rest of this section is devoted to the proof of Theorem A.5 in the case $p=1$. For $p>1$, the proof is very similar, except that instead of conditioning by the deviations of the extreme eigenvalues of $H_{N}$, we will condition on the deviations of extreme eigenvalues of the model at step $p-1$.

Proof of Theorem A.5 in the case $p=1$. We recall that we stick to the case $\beta=1$. Let $\gamma_{1}>0$ be fixed. As in the proof of Theorem 2.5, the exponential tightness is straightforward : for any $N \geq 1$,

$$
\left|\widetilde{\lambda_{\max }^{N}}\right| \leq K+\gamma_{1}
$$

Again, using e.g. Theorem D.4(a) and Corollary D.6 in [1], it is enough to show that, for any $x \in \mathbb{R}$,

$$
\begin{aligned}
\lim _{\delta \downarrow 0} \limsup _{N \rightarrow \infty} \frac{1}{N} \log \left(m_{N}^{1}\right)^{\otimes 2} & \left(\widetilde{\lambda_{\max }^{N}} \in[x-\delta, x+\delta]\right) \\
& =\lim _{\delta \downarrow 0} \liminf _{N \rightarrow \infty} \frac{1}{N} \log \left(m_{N}^{1}\right)^{\otimes 2}\left(\widetilde{\lambda_{\max }^{N}} \in[x-\delta, x+\delta]\right)=-L_{\gamma}^{(1)}(x) .
\end{aligned}
$$

For any $z$ which does not belong to the spectrum of $H_{N}$, one can write

$$
\operatorname{det}\left(z I_{N}-X_{N}\right)=\operatorname{det}\left(z I_{N}-H_{N}\right) \gamma_{1}\left(\frac{1}{\gamma_{1}}-\left(U_{1}^{(1)}\right)^{*}\left(z I_{N}-H_{N}\right)^{-1} U_{1}^{(1)}\right) .
$$

Therefore, $z$ is an eigenvalue of $X_{N}$ which is not an eigenvalue of $H_{N}$ if and only if

$$
\left(U_{1}^{(1)}\right)^{*}\left(z I_{N}-H_{N}\right)^{-1} U_{1}^{(1)}=\frac{1}{\gamma_{1}} .
$$

The Haar measure is invariant by unitary conjugation, so that if $H_{N}=U_{N} D_{N} U_{N}^{*}$, then $\left(U_{1}^{(1)}\right)^{*}\left(z I_{N}-H_{N}\right)^{-1} U_{1}^{(1)}$ and $\left(U_{1}^{(1)}\right)^{*}\left(z I_{N}-D_{N}\right)^{-1} U_{1}^{(1)}$ have the same law and one can assume in the sequel that $H_{N}$ is diagonal. Moreover, as $U_{1}^{(1)}$ is a column vector of a matrix distributed according to the Haar measure, the equation now reads

$$
v_{N}(z):=\sum_{i=1}^{N} \frac{1}{z-\lambda_{i}^{\left(H_{N}\right)}} \frac{g_{i}^{2}}{\sum_{i=1}^{N} g_{i}^{2}}=\frac{1}{\gamma_{1}},
$$

with $\left(g_{1}, \ldots, g_{N}\right)$ having distribution $P^{\otimes N}$. 
For any $\left(\lambda_{1}, \ldots, \lambda_{N}\right)$ and $\left(v_{1}, \ldots, v_{N}\right)$ such that $\sum_{i=1}^{N} v_{i}^{2}=1$ fixed, we define on $\left(\max _{i=1}^{N} \lambda_{i}, \infty\right)$, the function

$$
f_{\lambda, v}: z \mapsto \sum_{i=1}^{N} \frac{1}{z-\lambda_{i}} v_{i}^{2}
$$

This function is decreasing and continuous on $\left(\max _{i=1}^{N} \lambda_{i}, \infty\right)$, uniformly on $\left(v_{1}, \ldots, v_{N}\right)$ such that $\sum_{i=1}^{N} v_{i}^{2}=1$. Therefore, there exists a function $\varepsilon_{\lambda}$ going to zero at zero, such that for $z \in\left(\max _{i=1}^{N} \lambda_{i}, \infty\right), f_{\lambda}(z)=\frac{1}{\gamma_{1}}$ if and only if, for any $\delta>0$ small enough, for any $x \in[z-\delta, z+\delta], f_{\lambda}(x) \in\left[\frac{1}{\gamma_{1}}-\varepsilon_{\lambda}(\delta), \frac{1}{\gamma_{1}}+\varepsilon_{\lambda}(\delta)\right]$. Let $x>\mathrm{r}\left(\mu_{a} \boxplus \mu_{b}\right)$ be fixed. Let $y$ such that $\mathrm{r}\left(\mu_{a} \boxplus \mu_{b}\right) \leq y<x$ and set $\eta_{0}:=\frac{x-y}{4}$. For any $\eta<\eta_{0}$, similarly to the definition of $E_{N, \eta}^{y}$ in (3.6), we introduce

$$
\widetilde{E_{N, \eta}^{y}}:=\left\{\lambda_{\max }^{N} \in[y, y+\eta], \mathrm{d}\left(\hat{\mu}_{N}, \nu_{N}^{\beta}\right) \leq N^{-1 / 4}\right\}
$$

The analysis will be the same, except possibly for $y=\mathrm{r}\left(\mu_{a} \boxplus \mu_{b}\right)$. We have that for any $U \in \widetilde{E_{N, \eta}^{y}}, \lambda_{\max }^{N}=\lambda_{1}^{\left(H_{N}\right)} \in[y, y+\eta]$. Therefore, if we denote by

$$
\widetilde{v_{N}}(x, y):=\frac{1}{x-y} \frac{g_{1}^{2}}{\sum_{i=1}^{N} g_{i}^{2}}+\sum_{i=2}^{N} \frac{1}{x-\lambda_{i}^{\left(H_{N}\right)}} \frac{g_{i}^{2}}{\sum_{i=1}^{N} g_{i}^{2}}
$$

then

$$
\left|\widetilde{v_{N}}(x, y)-v_{N}(x)\right| \leq \frac{\left|y-\lambda_{1}^{\left(H_{N}\right)}\right|}{(x-y)\left(x-\lambda_{1}^{\left(H_{N}\right)}\right)} \leq \frac{\eta}{\eta_{0}^{2}}
$$

Therefore, for any $\eta<\eta_{0}$, there exists a continuous function $\varepsilon_{\eta}$ going to zero at zero such that, for any $\delta \leq \eta$ and $N$ large enough,

$$
\begin{aligned}
& \left(m_{N}^{1}\right)^{\otimes 2}\left(\left\{\widetilde{\lambda_{\max }^{N}} \in[x-\delta, x+\delta]\right\} \cap \widetilde{\mathrm{E}_{N, \eta}^{y}}\right) \\
& \quad=P^{\otimes N} \otimes m_{N}^{1}\left(\left\{\widetilde{v^{N}}(x, y) \in\left[\frac{1}{\gamma_{1}}-\varepsilon(\delta), \frac{1}{\gamma_{1}}+\varepsilon(\delta)\right]\right\} \cap \widetilde{\mathrm{E}_{N, \eta}^{y}}\right) \\
& \quad=P^{\otimes N} \otimes m_{N}^{1}\left(\widetilde{v^{N}}(x, y) \in\left[\frac{1}{\gamma_{1}}-\varepsilon(\delta), \frac{1}{\gamma_{1}}+\varepsilon(\delta)\right] \widetilde{\mathrm{E}_{N, \eta}^{y}}\right) m_{N}^{1}\left(\widetilde{\mathrm{E}_{N, \eta}^{y}}\right)
\end{aligned}
$$

The probability measure on the right handside is $P^{\otimes N} \otimes m_{N}^{1}$ because $\widetilde{v^{N}}(x, y)$ can be seen as a function of $U$ of law $m_{N}^{1}$ and of $\left(g_{1}, \ldots, g_{N}\right)$ of law $P^{\otimes N}$.

If we assume that $\eta, \delta<\frac{|x-y|}{4}$ and for all $i \in \mathbb{N}^{*}, \lambda_{i} \leq y+\eta$, one can choose $\varepsilon_{\lambda}$ uniformly in $\left(\lambda_{1}, \ldots, \lambda_{N}\right)$. Now, let $U \in \widetilde{\mathrm{E}_{N, \eta}^{y}}$ be chosen. We denote by $\lambda_{1}^{N}:=y$ and for any $2 \leq i \leq N, \lambda_{i}^{N}:=\lambda_{i}^{\left(H_{N}\right)}$. Then $\frac{1}{N} \sum_{i=1}^{N} \delta_{\lambda_{i}^{N}}$ converges to $\mu_{a} \boxplus \mu_{b}$ and $\max _{i=1}^{N} \lambda_{i}^{N}=y$. By Proposition A.6, if $G_{\mu_{a} \boxplus \mu_{b}}(x) \leq \frac{1}{\gamma_{1}}$, we have

$$
\lim _{\delta \downarrow 0} \liminf _{N \rightarrow \infty} \frac{1}{N} \log P^{\otimes N}\left(\widetilde{v_{N}}(x) \in\left[\frac{1}{\gamma_{1}}-\varepsilon_{\eta}(\delta), \frac{1}{\gamma_{1}}+\varepsilon_{\eta}(\delta)\right] \widetilde{\mid \mathrm{E}_{N, \eta}^{y}}\right)=-T_{x, y}^{+}\left(\frac{1}{\gamma_{i}}\right),
$$

so that

$$
\lim _{\delta \downarrow 0} \liminf _{N \rightarrow \infty} \frac{1}{N} \log \left(m_{N}^{1}\right)^{\otimes 2}\left(\widetilde{\lambda_{\max }^{N}} \in[x-\delta, x+\delta]\right) \geq-T_{x, y}^{+}\left(\frac{1}{\gamma_{1}}\right)+\lim _{N \rightarrow \infty} \frac{1}{N} \log m_{N}^{1}\left(\widetilde{E_{N, \eta}^{y}}\right) .
$$

Taking the limit of the right hand-side as $\eta$ goes to zero, we get using Theorem 2.5 that

$$
\lim _{\delta \downarrow 0} \lim _{N \rightarrow \infty} \frac{1}{N} \log \left(m_{N}^{1}\right)^{\otimes 2}\left(\widetilde{\lambda_{\max }^{N}} \in[x-\delta, x+\delta]\right) \geq-T_{x, y}^{+}\left(\frac{1}{\gamma_{1}}\right)-I^{1}(y) \geq-L_{\gamma}^{(1)}(x),
$$

where the last inequality was obtained by optimizing on $y$. 
Assume now that $\mathrm{r}:=\mathrm{r}\left(\mu_{a} \boxplus \mu_{b}\right)<x<K_{\mu_{a} \boxplus \mu_{b}}\left(\frac{1}{\gamma_{1}}\right)$. Similarly to (3.6), we define, for $y<\mathrm{I}\left(\mu_{a} \boxplus \mu_{b}\right)$

$$
\mathrm{E}_{N, \eta}^{y,-}:=\left\{\lambda_{\min }^{N} \in[y, y+\eta], \lambda_{\max }^{N} \in[\mathrm{r}-\eta, \mathrm{r}+\eta], \mathrm{d}\left(\hat{\mu}_{N}, \nu_{N}^{1}\right) \leq N^{-1 / 4}\right\},
$$

and

$$
v_{N,-}(x):=\sum_{i=1}^{N-1} \frac{1}{x-\lambda_{i}^{\left(H_{N}\right)}} \frac{g_{i}^{2}}{\sum_{i=1}^{N} g_{i}^{2}}+\frac{1}{x-y} \frac{g_{N}^{2}}{\sum_{i=1}^{N} g_{i}^{2}} .
$$

For $\eta$ small enough and $\delta \leq \eta$, we can then write as above

$$
\begin{aligned}
\left(m_{N}^{1}\right)^{\otimes 2} \widetilde{\lambda_{\max }^{N}} & \in[x-\delta, x+\delta]) \geq\left(m_{N}^{1}\right)^{\otimes 2}\left(\widetilde{\lambda_{\max }^{N}} \in[x-\delta, x+\delta] \cap \mathrm{E}_{N, \eta}^{y,-}\right) \\
& =P^{\otimes n} \otimes m_{N}^{1}\left(v_{N,-}(x) \in\left[\frac{1}{\gamma_{1}}-\varepsilon_{\eta}(\delta), \frac{1}{\gamma_{1}}+\varepsilon_{\eta}(\delta)\right] \cap E_{N, \eta}^{y,-}\right) \\
= & P^{\otimes n} \otimes m_{N}^{1}\left(v_{N,-}(x) \in\left[\frac{1}{\gamma_{1}}-\varepsilon_{\eta}(\delta), \frac{1}{\gamma_{1}}+\varepsilon_{\eta}(\delta)\right] \mid E_{N, \eta}^{y,-}\right) m_{N}^{1}\left(E_{N, \eta}^{y,-}\right)
\end{aligned}
$$

In this case, by Proposition A.6,

$$
\lim _{\delta \downarrow 0} \liminf _{N \rightarrow \infty} \frac{1}{N} \log P^{\otimes N}\left(v_{N,-}(x) \in\left[\frac{1}{\gamma_{1}}-\varepsilon_{\eta}(\delta), \frac{1}{\gamma_{1}}+\varepsilon_{\eta}(\delta)\right] \mid \mathrm{E}_{N, \eta}^{y,-}\right)=-T_{x, y}^{-}\left(\frac{1}{\gamma_{1}}\right),
$$

so that

$$
\lim _{\delta \downarrow 0} \liminf _{N \rightarrow \infty} \frac{1}{N} \log \left(m_{N}^{1}\right)^{\otimes 2}\left(\widetilde{\lambda_{\max }^{N}} \in[x-\delta, x+\delta]\right)=-T_{x, y}^{-}\left(\frac{1}{\gamma_{1}}\right)+\liminf _{N \rightarrow \infty} \frac{1}{N} \log m_{N}^{1}\left(E_{N, \eta}^{y,-}\right) .
$$

The last step to prove the lower bound in this case is to check

$$
\lim _{\eta \downarrow 0} \liminf _{N \rightarrow \infty} \frac{1}{N} \log m_{N}^{1}\left(E_{N, \eta}^{y,-}\right) \geq-I_{\min }^{1}(y) .
$$

Then, taking the limit as $\eta$ goes to zero in (A.13) and optimizing in $y$ gives the required lower bound.

We now prove (A.14). Similarly to Lemma 4.2 and 5.1 (by symmetry between the smallest and largest eigenvalue), one can show that there exists a unique $\theta_{y} \leq 0$ such that, for any $\eta>0$ and $N$ large enough,

$$
m_{N}^{1, \theta_{y}}\left(\lambda_{\min }^{N} \in[y-\eta, y+\eta], \mathrm{d}\left(\hat{\mu}_{N}, \nu_{N}^{1}\right) \leq N^{-1 / 4}\right) \geq \frac{2}{3} .
$$

Applying Corollary A.4, as $\theta_{y} \leq 0$, we have that, for any $\eta>0$ and $N$ large enough,

$$
m_{N}^{1, \theta_{y}}\left(\lambda_{\max }^{N} \in[\mathrm{r}-\eta, \mathrm{r}+\eta]\right) \geq \frac{2}{3},
$$

so that, for any $\eta>0$ and $N$ large enough,

$$
m_{N}^{1, \theta_{y}}\left(\mathrm{E}_{N, \eta}^{y,-}\right) \geq \frac{1}{3}
$$

With this ingredient, the proof of (A.14) goes as in the proof of Proposition 2.4:

$$
\begin{aligned}
m_{N}^{1}\left(\mathrm{E}_{N, \eta}^{y,-}\right) & =\mathbb{E}_{m_{N}^{1}}\left(1_{\mathrm{E}_{N, \eta}^{y,-}} \frac{I_{N}^{1}\left(\theta_{y}, H\right)}{I_{N}^{1}\left(\theta_{y}, H\right)}\right) \\
& \geq \inf _{U \in \mathrm{E}_{N, \eta}^{y,-}} \frac{1}{I_{N}^{1}\left(\theta_{y}, A+U B U^{*}\right)} I_{N}^{1}\left(\theta_{y}, A\right) I_{N}^{1}\left(\theta_{y}, B\right) m_{N}^{1, \theta_{y}}\left(\mathrm{E}_{N, \eta}^{y,-}\right),
\end{aligned}
$$


so that, using again Lemma 3.4, we get:

$$
\lim _{\eta \downarrow 0} \liminf _{N \rightarrow \infty} \frac{1}{N} \log m_{N}^{1}\left(\mathrm{E}_{N, \eta}^{y,-}\right) \geq-I_{\min }^{1}\left(\theta_{y}, y\right)-\lim _{\eta \downarrow 0} g_{\theta_{y}}(\eta)=-I_{\min }^{1}(y) .
$$

The strategy to get the upper bound is similar: we know that, for $N \geq 1, \lambda_{\max }^{N} \in$ $[-K, K]$ and $\lambda_{\min }^{N} \in[-K, K]$. For any $\delta>0$, there exists $p \in \mathbb{N}^{*}$ and $\rho_{1}, \ldots, \rho_{p}$ such that

$$
[-K, K] \subset \cup_{i=1}^{p}\left[\rho_{i}-\delta, \rho_{i}+\delta\right] .
$$

Assume that $G_{\mu_{a} \boxplus \mu_{b}}(x) \leq \frac{1}{\gamma_{1}}$.

$$
\begin{aligned}
\left.\left(m_{N}^{1}\right)^{\otimes 2} \widetilde{\lambda_{\max }^{N}} \in[x-\delta, x+\delta]\right) & \left.\leq\left(m_{N}^{1}\right)^{\otimes 2} \widetilde{\lambda_{\max }^{N}} \in[x-\delta, x+\delta] \cap\left\{\mathrm{d}\left(\hat{\mu}_{N}, \nu_{N}^{1}\right) \leq N^{-1 / 4}\right\}\right) \\
& +m_{N}^{1}\left(\mathrm{~d}\left(\hat{\mu}_{N}, \nu_{N}^{1}\right)>N^{-1 / 4}\right) \\
& \left.\leq \sum_{i=1}^{p}\left(m_{N}^{1}\right)^{\otimes 2} \widetilde{\lambda_{\max }^{N}} \in[x-\delta, x+\delta] \cap \mathrm{E}_{N, \delta}^{\rho_{i}}\right) \\
& +m_{N}^{1}\left(\mathrm{~d}\left(\hat{\mu}_{N}, \nu_{N}^{1}\right)>N^{-1 / 4}\right) .
\end{aligned}
$$

We then use Lemma 3.3 to get rid of the last term and apply the same strategy as before, combining the relation (A.12) and Proposition A.6 for the main term.

Assume now that $G_{\mu_{a} \boxplus \mu_{b}}(x)>\frac{1}{\gamma_{1}}$. We apply the very same strategy with $\mathrm{E}_{N, \delta}^{\rho_{i},-}$ instead of $\mathrm{E}_{N, \delta}^{\rho_{i}}$ and the bound (A.14).

\section{References}

[1] Greg W. Anderson, Alice Guionnet, and Ofer Zeitouni, An introduction to random matrices, Cambridge Studies in Advanced Mathematics, vol. 118, Cambridge University Press, Cambridge, 2010. MR-2760897

[2] Fanny Augeri, Large deviations principle for the largest eigenvalue of Wigner matrices without Gaussian tails, Electron. J. Probab. 21 (2016), Paper No. 32, 49. MR-3492936

[3] Fanny Augeri, On the large deviations of traces of random matrices, Ann. Inst. Henri Poincaré Probab. Stat. 54 (2018), no. 4, 2239-2285. MR-3865672

[4] Zhigang Bao, László Erdős, and Kevin Schnelli, Local law of addition of random matrices on optimal scale, Comm. Math. Phys. 349 (2017), no. 3, 947-990. MR-3602820

[5] Serban Teodor Belinschi, A note on regularity for free convolutions, Ann. Inst. H. Poincaré Probab. Statist. 42 (2006), no. 5, 635-648. MR-2259979

[6] Serban Teodor Belinschi, The Lebesgue decomposition of the free additive convolution of two probability distributions, Probab. Theory Related Fields 142 (2008), no. 1-2, 125-150. MR-2413268

[7] Serban Teodor Belinschi, Hari Bercovici, Mireille Capitaine, and Maxime Février, Outliers in the spectrum of large deformed unitarily invariant models, Ann. Probab. 45 (2017), no. 6A, 3571-3625. MR-3729610

[8] Gérard Ben Arous, Amir Dembo, and Alice Guionnet, Aging of spherical spin glasses, Probab. Theory Related Fields 120 (2001), no. 1, 1-67. MR-1856194

[9] Gérard Ben Arous and Alice Guionnet, Large deviations for Wigner's law and Voiculescu's non-commutative entropy, Probab. Theory Related Fields 108 (1997), no. 4, 517-542. MR1465640

[10] Florent Benaych-Georges, Alice Guionnet, and Mylène Maïda, Large deviations of the extreme eigenvalues of random deformations of matrices, Probab. Theory Related Fields 154 (2012), no. 3-4, 703-751. MR-3000560

[11] Bernard Bercu, Fabrice Gamboa, and Alain Rouault, Large deviations for quadratic forms of stationary Gaussian processes, Stochastic Process. Appl. 71 (1997), no. 1, 75-90. MR1480640 
LDP for the largest eigenvalue of the sum of random matrices

[12] Charles Bordenave and Pietro Caputo, A large deviation principle for Wigner matrices without Gaussian tails, Ann. Probab. 42 (2014), no. 6, 2454-2496. MR-3265172

[13] Mireille Capitaine and Catherine Donati-Martin, Spectrum of deformed random matrices and free probability, Advanced topics in random matrices, Panor. Synthèses, vol. 53, Soc. Math. France, Paris, 2017, pp. 151-190. MR-3792626

[14] Benoît Collins and Camille Male, The strong asymptotic freeness of Haar and deterministic matrices, Ann. Sci. Éc. Norm. Supér. (4) 47 (2014), no. 1, 147-163. MR-3205602

[15] Benoît Collins and Piotr Śniady, New scaling of Itzykson-Zuber integrals, Ann. Inst. H. Poincaré Probab. Statist. 43 (2007), no. 2, 139-146. MR-2303115

[16] Catherine Donati-Martin and Mylène Maïda, Large deviations for the largest eigenvalue of an Hermitian Brownian motion, ALEA Lat. Am. J. Probab. Math. Stat. 9 (2012), no. 2, 501-530. MR-3069375

[17] Anne Fey, Remco van der Hofstad, and Marten J. Klok, Large deviations for eigenvalues of sample covariance matrices, with applications to mobile communication systems, Adv. in Appl. Probab. 40 (2008), no. 4, 1048-1071. MR-2488532

[18] Benjamin Groux, Asymptotic freeness for rectangular random matrices and large deviations for sample convariance matrices with sub-Gaussian tails, Electron. J. Probab. 22 (2017), Paper No. 53, 40. MR-3666016

[19] Alice Guionnet and Jonathan Husson, Large deviations for the largest eigenvalue of Rademacher matrices, to appear in Annals of Probab. (2020), https://arxiv.org/abs/1810. 01188.

[20] Alice Guionnet and Mylène Maïda, A Fourier view on the $R$-transform and related asymptotics of spherical integrals, J. Funct. Anal. 222 (2005), no. 2, 435-490. MR-2132396

[21] Alice Guionnet and Ofer Zeitouni, Large deviations asymptotics for spherical integrals, J. Funct. Anal. 188 (2002), no. 2, 461-515. MR-1883414

[22] Vladislav Kargin, A concentration inequality and a local law for the sum of two random matrices, Probab. Theory Related Fields 154 (2012), no. 3-4, 677-702. MR-3000559

[23] Mylène Maïda, Large deviations for the largest eigenvalue of rank one deformations of Gaussian ensembles, Electron. J. Probab. 12 (2007), 1131-1150. MR-2336602

[24] Elizabeth S. Meckes and Mark W. Meckes, Concentration and convergence rates for spectral measures of random matrices, Probab. Theory Related Fields 156 (2013), no. 1-2, 145-164. MR-3055255

[25] L. Pastur and V. Vasilchuk, On the law of addition of random matrices, Comm. Math. Phys. 214 (2000), no. 2, 249-286. MR-1796022

[26] Roland Speicher, Free convolution and the random sum of matrices, Publ. Res. Inst. Math. Sci. 29 (1993), no. 5, 731-744. MR-1245015

[27] Dan Voiculescu, Limit laws for random matrices and free products, Invent. Math. 104 (1991), no. 1, 201-220. MR-1094052

Acknowledgments. The idea to tilt measures by the spherical integral came out magically from a discussion with M. Potters in UCLA in 2017 and we wish to thank him for this beautiful inspiration. We also benefited from many discussions with J. Husson and F. Augeri with whom one of the author is working on a companion project on Wigner matrices. We also thank Benjamin McKenna for pointing out a gap in the proof of Proposition 3.1 and anonymous referees for very useful remarks in particular on the Appendix. Finally, we are very grateful for stimulating discussions with O. Zeitouni and N. Cook. 